\title{
Drosophila Neurexin IV Interacts with Roundabout and Is Required for Repulsive Midline Axon Guidance
}

\author{
Swati Banerjee, ${ }^{1}$ Kevin Blauth, ${ }^{2}$ Kimberly Peters, ${ }^{3}$ Stephen L. Rogers, ${ }^{3}$ Alan S. Fanning, ${ }^{1}$ and Manzoor A. Bhat ${ }^{1,2,4,5}$ \\ ${ }^{1}$ Department of Cell and Molecular Physiology, ${ }^{2}$ Curriculum in Neurobiology, ${ }^{3}$ Department of Biology, Carolina Center for Genome Sciences, Lineberger \\ Cancer Center, ${ }^{4}$ University of North Carolina Neuroscience Center, and ${ }^{5}$ Neurodevelopmental Disorders Research Center, University of North Carolina \\ School of Medicine Chapel Hill, Chapel Hill, North Carolina 27599-7545
}

\begin{abstract}
Slit/Roundabout (Robo) signaling controls midline repulsive axon guidance. However, proteins that interact with Slit/Robo at the cell surface remain largely uncharacterized. Here, we report that the Drosophila transmembrane septate junction-specific protein Neurexin IV (Nrx IV) functions in midline repulsive axon guidance. Nrx IV is expressed in the neurons of the developing ventral nerve cord, and $n r x$ $I V$ mutants show crossing and circling of ipsilateral axons and fused commissures. Interestingly, the axon guidance defects observed in $n r x I V$ mutants seem independent of its other binding partners, such as Contactin and Neuroglian and the midline glia protein Wrapper, which interacts in trans with Nrx IV. $n r x I V$ mutants show diffuse Robo localization, and dose-dependent genetic interactions between $n r x$ $I V / r o b o$ and $n r x I V / s l i t$ indicate that they function in a common pathway. In vivo biochemical studies reveal that Nrx IV associates with Robo, Slit, and Syndecan, and interactions between Robo and Slit, or Nrx IV and Slit, are affected in $n r x I V$ and robo mutants, respectively. Coexpression of Nrx IV and Robo in mammalian cells confirms that these proteins retain the ability to interact in a heterologous system. Furthermore, we demonstrate that the extracellular region of Nrx IV is sufficient to rescue Robo localization and axon guidance phenotypes in $n r x I V$ mutants. Together, our studies establish that Nrx IV is essential for proper Robo localization and identify Nrx IV as a novel interacting partner of the Slit/Robo signaling pathway.
\end{abstract}

\section{Introduction}

A common organizational principle among all organisms is that the two halves of the CNS are interconnected by commissures in which interneurons project across the midline (ML). In Drosophila CNS, many of these interneurons project axons across the ML of the ventral nerve cord (VNC) in one of two distinct tracts: the anterior commissure (AC) or the posterior commissure (PC), each of which connects the opposing sides of the CNS (Goodman and Doe, 1993; Jacobs, 2000). Collectively, axons extending from multiple populations of contralaterally and ipsilaterally projecting interneurons follow stereotypic trajectories forming the conspicuous orthogonal array that represents the Drosophila VNC. Specialized midline glia (MG) attract or repulse axons by secreting ligands (Garbe and Bashaw, 2004, 2007; O'Donnell et al., 2009). Netrin A and B are attractive glia-derived ligands that interact with their respective neuronal receptors, Frazzled and Unc5, respectively (Harris et al., 1996; Kolodziej et al., 1996; Mitchell et al., 1996; Keleman and Dickson, 2001; Brankatschk

Received Dec. 14, 2009; revised Feb. 26, 2010; accepted March 14, 2010.

This work was supported by National Institutes of Health Grants NS050356 and GM063074 (M.A.B.) and funds from the State of North Carolina (M.A.B.). We thank Drs. G. Bashaw, D. Van Vactor, T. Kidd, S. Crews, C. FaivreSarrailh, J. Lincecum, J. Noordermeer, and G. Vorbruggen for reagents and Drs. S. Crews, S. Wheeler, and M. McMurray and members of the Bhat laboratory for valuable discussions. We also thank the Developmental Studies Hybridoma Bank, University of lowa, for monoclonal antibodies and the Bloomington Stock Center for fly reagents.

Correspondence should be addressed to Dr. Manzoor A. Bhat, University of North Carolina Neuroscience Center, Neuroscience Research Building \#5109, University of North Carolina School of Medicine, Chapel Hill, NC 27599-7545. E-mail:Manzoor_Bhat@med.unc.edu.

DOI:10.1523/JNEUROSCI.6187-09.2010

Copyright $\odot 2010$ the authors $\quad 0270-6474 / 10 / 305653-15 \$ 15.00 / 0$ and Dickson, 2006; Garbe and Bashaw, 2007; Yang et al., 2009). The repulsive axon guidance pathway comprises the glial ligand Slit and the Robo family of transmembrane receptors (Seeger et al., 1993; Kidd et al., 1998, 1999; Battye et al., 1999; Rajagopalan et al., 2000; Simpson et al., 2000). Slit, Netrin, and their respective neuronal receptors play critical roles in patterning axonal connections in the developing nervous system ML across species. Recent studies have shown that Slit and Netrin influence ML axon crossing by both independent and interdependent signaling by Robo and Frazzled (Garbe and Bashaw, 2007; Yang et al., 2009).

The high fidelity of Slit/Robo interactions is evolutionarily conserved; Drosophila Slit binds with mammalian Robo and vice versa (Brose et al., 1999). How the interactions between secreted Slit and Robo transduce signals intracellularly, and the proteins involved in Slit/Robo interactions at the cell surface, remain unclear. Recently, the heparan sulfate proteoglycan (HSPG) Syndecan (Sdc) was shown to form a molecular complex with Slit/ Robo. $s d c$ mutants displayed axon guidance defects, but little is known regarding the mechanisms that ensure Robo localization and stabilization and its interactions with Slit at the cell surface (Johnson et al., 2004; Steigemann et al., 2004; Chanana et al., 2009). Studies by Dickson and colleagues have shown that Commissureless controls axon guidance across the Drosophila midline by regulating trafficking and therefore the cell surface levels of the Robo protein (Keleman et al., 2002, 2005). A better understanding of the intracellular molecular events occurring within axons has emerged from Drosophila and mammalian studies (Fritz and VanBerkum, 2000, 2002; Fan et al., 2003; Yang and Bashaw, 
2006). It is conceivable that additional coreceptors or accessory proteins participate in presenting and/or stabilizing the secreted axon guidance factors for efficient and controlled signal transduction to allow the growing axons to navigate to their final destination.

Recent studies from our laboratory and others revealed that Neurexin IV (Nrx IV) is expressed in ML neurons and interacts in trans with MG protein Wrapper to organize the neuron-glial scaffold (Stork et al., 2009; Wheeler et al., 2009). Here we report novel and distinct functions of Nrx IV in axon guidance at the embryonic ML that are independent of its binding partners, Contactin, Neuroglian, and Wrapper. Our studies establish that Nrx IV is essential for proper Robo localization and repulsive axon guidance and thus identify Nrx IV as a novel interacting component of the Robo/Slit signaling pathway.

\section{Materials and Methods}

Drosophila stocks and molecular biology reagents. The Drosophila mutants used in this study are: $n r x I V^{4304}$ (Baumgartner et al., 1996), robo ${ }^{\text {GA285 }}$ (Kidd et al., 1998), slit ${ }^{2}$ (Rothberg et al., 1990), $\mathrm{nrg}^{1}$, cont ${ }^{\text {ex956 }}$ (FaivreSarrailh et al., 2004), wrapper ${ }^{175}$ (Noordermeer et al., 1998), $s d c^{10608}$, $D f 48^{u b i-S a r a}$ (Johnson et al., 2004), $f r a^{3}, f r a^{4}$, and net ${ }^{A, B \Delta}$ (Andrews et al., 2008). The Gal4 and UAS lines used are as follows: UAS-nrx IV ${ }^{m y c}, U A S$ nrx IV ${ }^{m y c \Delta C T}$, UAS-nrx IV ${ }^{m y c \Delta N T}$ (this study), UAS-tau GFP (Brand, 1995); elav-Gal4, sim-Gal4, repo-Gal4, ap-Gal4, and nrx IV:GFP (CA06597) (Buszczak et al., 2007) obtained from Bloomington Stock Center and the FlyTrap project. For expression of robo and slit in mammalian HEK 293 and Chinese hamster ovary (CHO) cells, the respective cDNAs were cloned into pcDNA3.1 (Invitrogen) and expressed using standard cell culture experiments. Expression of $n r x I V$ and cont in mammalian cells has been previously reported (Faivre-Sarrailh et al., 2004).

Immunofluorescence of embryos and mammalian cells. Antibody staining of embryos was done as previously described (Banerjee et al., 2006). Primary antibodies used were as follows: rabbit anti-Nrx IV (1:500), rat anti-Wrapper (1:250; Wheeler et al., 2009), chicken anti-GFP (1:500, Invitrogen), monoclonals including anti-Fasciclin II (anti-Fas II) (ID4, 1:250), BP102 (1:500), anti-Robo (13C9, 1:10), and anti-Slit (C555.6D, 1:50), which were obtained from the Developmental Studies Hybridoma Bank, University of Iowa. Isotype-specific and fluorescent secondary antibodies Alexa 488, 568, and 647 were obtained from Jackson Immunochemicals and Invitrogen. The Zenon mouse IgG1 labeling kit (Invitrogen) was used for colocalization studies of Fas II and Robo according to the manufacturer's instructions.

CHO K1 and HEK 293 cells were obtained from the Lineberger Comprehensive Cancer Center (University of North Carolina Chapel Hill, Chapel Hill, NC) and maintained in DMEM supplemented with $10 \%$ fetal bovine serum, penicillin, and streptomycin in a humidified incubator at $37^{\circ} \mathrm{C}$ with $5 \% \mathrm{CO}_{2}$. Cells $\left(1.0 \times 10^{6}\right)$ were plated on coverslips in 60 $\mathrm{mm}$ dishes, and after $24 \mathrm{~h}$ they were transfected with Fugene6 (Roche Diagnostics) according to the manufacturer's instructions. Following a $48 \mathrm{~h}$ incubation, cells were washed twice in PBS and fixed in a freshly prepared $4 \%$ solution of formaldehyde in PBS. Cells were then washed with PBS, extracted with 2\% Triton X-100 (Sigma-Aldrich) in PBS for 10 min, washed again, and blocked in $5 \%$ normal donkey serum (Jackson ImmunoResearch) in PBS for $2 \mathrm{~h}$ at $25^{\circ} \mathrm{C}$. Cells were stained with primary antibody diluted in 5\% bovine serum albumin (BSA Fraction V, Sigma-Aldrich) in PBS overnight at $4^{\circ} \mathrm{C}$. Antibody dilutions were identical to those used for Drosophila embryos. Following primary antibody incubation, cells were washed three times for 15 min each with PBS/BSA and subsequently incubated with 1:100 dilution of affinity-purified Cy2conjugated donkey anti-rabbit (Nrx IV) and a 1:500 dilution of Cy3conjugated donkey anti-mouse (Jackson ImmunoResearch) for $2 \mathrm{~h}$ at $25^{\circ} \mathrm{C}$. Cells were washed as described above and mounted in Mowiol (Calbiochem) with 1\% n-propylgallate (Sigma-Aldrich). Wherever necessary, the immunofluorescence images were captured under identical settings with a Z-step of $0.25 \mu \mathrm{m}$ for embryos and $0.2 \mu \mathrm{m}$ for $\mathrm{CHO}$ cells on a Bio-Rad Radiance confocal microscope and processed with Adobe PhotoShop software.

Statistical analyses of axon guidance defects. Four hundred abdominal segments from 50 embryos of various genotypes at stage 16 were examined for aberrant axon crossings at the ML. Data are presented as average number of ML crossings per embryo for each genotype. All genotypes were analyzed using ANOVA and error bars indicate SEM. ${ }^{*}$ indicates a $p$ value of $<0.05,{ }^{* *}$ indicates a $p$ value of $<0.01$, and ${ }^{* *}$ indicates a $p$ value of $<0.001$.

Collection of homozygous mutant embryos. nrx IV, robo, and slit mutants were balanced with balancer chromosomes carrying twi-GFP, and the respective homozygous mutant embryos were automatically sorted with the Complex Object Parametric Analyzer and Sorter (COPAS) Select embryo sorter (Union Biometrica) by their lack of GFP expression according to published procedures (Furlong et al., 2001). The efficiency and quality of the sorted non-GFP mutant embryos were visually inspected for any GFP-positive embryos by using a GFP microscope and found to be close to $100 \%$, and if any contaminating GFP-positive embryos were seen, they were manually removed. Wild-type Canton S embryos were identically processed through the sorting procedure and used as controls. All protein estimations in lysates were performed using the DC Bio-Rad assay.

Drosophila S2 cell culture and RNAi. Drosophila S2 cell culture and RNAi were performed as described in the study by Rogers and Rogers (2008). Templates for in vitro transcription were generated by PCR with the primers encoding the T7 promoter sequence upstream of the following: slit (5'-TGCTCATCTTGCAGTGGAAC-3' and 5'CTTCATGCAGCCCTTAAAGC-3'). The efficiency of RNAi was determined by immunoblotting of S2 lysates with the Slit monoclonal antibody C555.6D. Overall protein amounts loaded for immunoblotting were normalized after estimation and verified using anti- $\beta$ tubulin antibodies.

Immunoprecipitation and immunoblotting. Embryos of desired genotypes were homogenized using a glass homogenizer in a weight/volume ratio of 1:4 in ice-cold lysis buffer containing 50 mM HEPES, pH 7.2, 100 mм NaCl, $1 \mathrm{~mm} \mathrm{MgCl}_{2}, 1 \mathrm{~mm} \mathrm{CaCl}_{2}$ and $1 \% \mathrm{NP}-40$ with protease inhibitors. HEK 293 cells were washed with PBS and lysed in $250 \mu$ l of lysis buffer. The lysates were kept on ice for $30 \mathrm{~min}$ and centrifuged at $15,000 \times g$ for $30 \mathrm{~min}$ at $4^{\circ} \mathrm{C}$, followed by recentrifugation, and used subsequently for immunoprecipitation (IP). For each IP reaction, $100 \mu \mathrm{l}$ of supernatant was precleared with Protein A or G beads followed by incubation with primary antibodies at 1:20 dilution (anti-Nrx IV, antiSlit, anti-Robo, and anti-Sdc) for $8 \mathrm{~h}$ at $4^{\circ} \mathrm{C}$. The supernatant-antibody mix was incubated with $25 \mu$ l of prewashed Protein A or G beads for $2 \mathrm{~h}$ at $4^{\circ} \mathrm{C}$. The beads were then washed three times in PBS followed by elution of the immunocomplexes in $30 \mu \mathrm{l}$ of PBS/SDS buffer and resolved by SDS-PAGE for immunoblotting with respective antibodies. IP data presented below (see Figs. $6 B-G, L, M, 7 K-P$ ) were performed using identical experimental conditions.

\section{Results}

Ipsilateral axons cross the midline and commissures fail to separate in $n r x I V$ mutants

We have previously established the role of Nrx IV in septate junction (SJ) organization (Baumgartner et al., 1996; Faivre-Sarrailh et al., 2004; Banerjee et al., 2006, 2008). However, apart from this traditional role of Nrx IV in junctional organization and function, additional roles of Nrx IV in the developmental organization of the embryonic ML have begun to emerge (Stork et al., 2009; Wheeler et al., 2009). In the present study, we analyzed the precise subcellular localization of Nrx IV in the developing CNS of wild-type embryos together with two well known monoclonal antibody markers, ID4 (against Fas II) and BP102. Immunostaining of wild-type $(+/+)$ embryos from stage 12 through late 16/17 with antibodies against Nrx IV, Fas II, and BP102 (Fig. $1 A, C, E, G, I)$ shows that at stage $12 \mathrm{Nrx}$ IV is expressed in the central group of cells also stained by Fas II, namely, the cluster of 


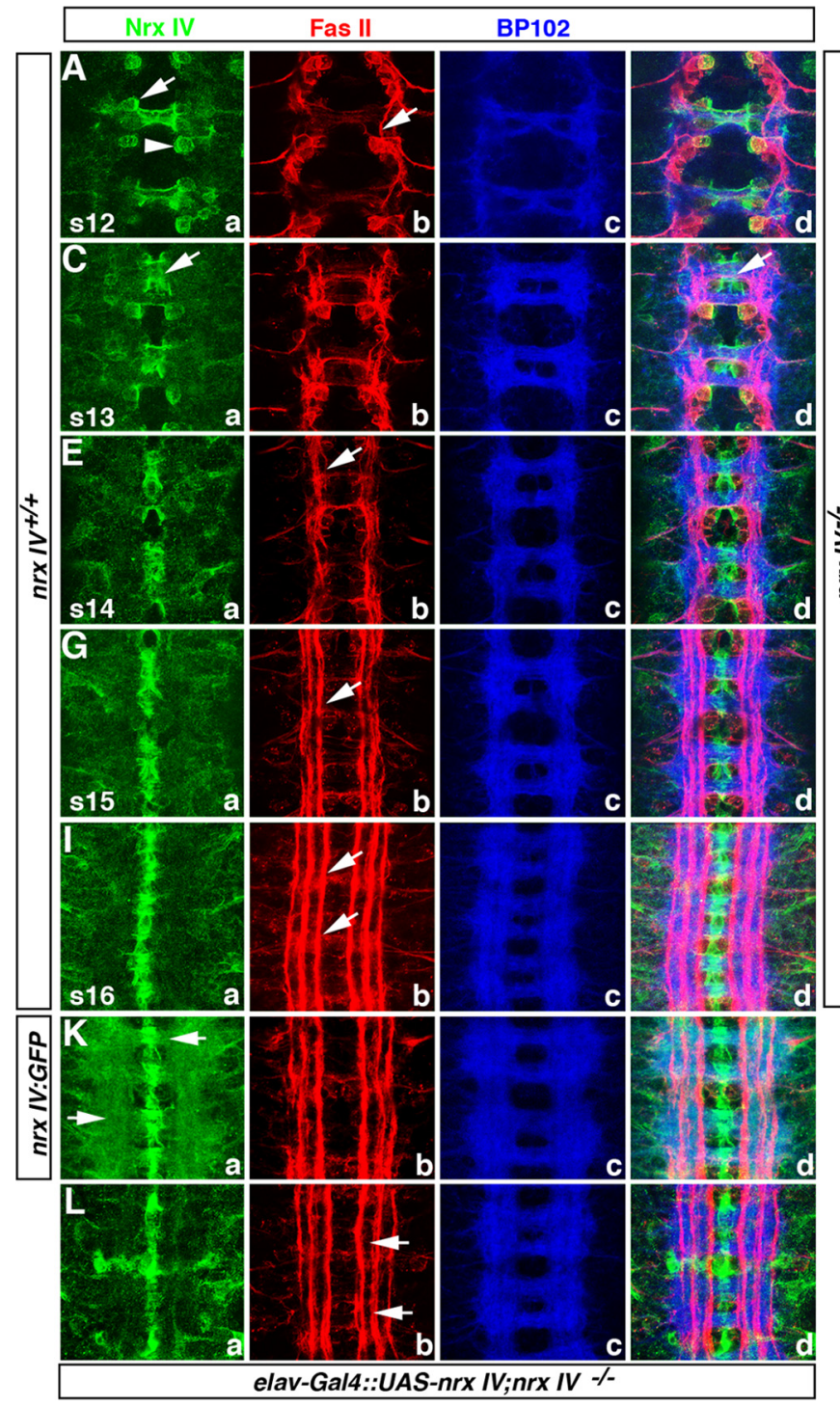

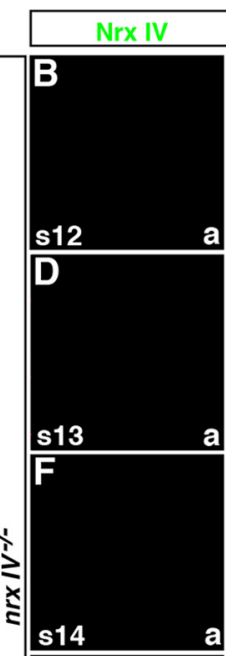

Fas II BP102
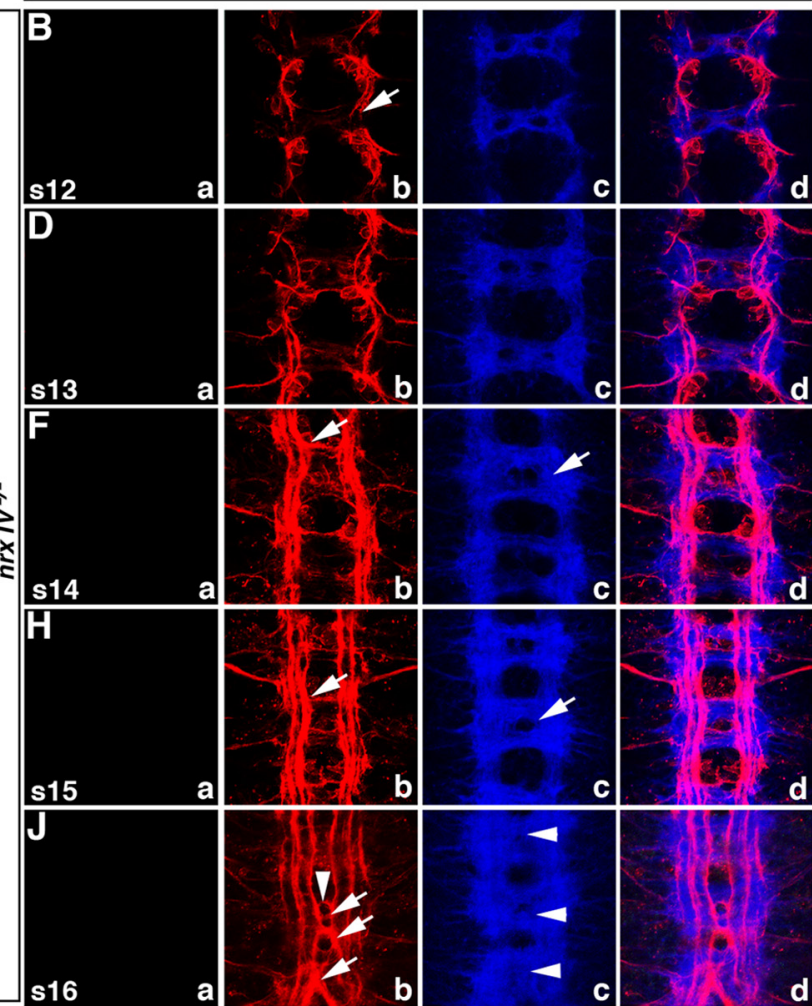

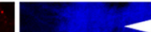

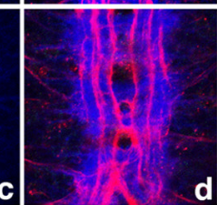

M

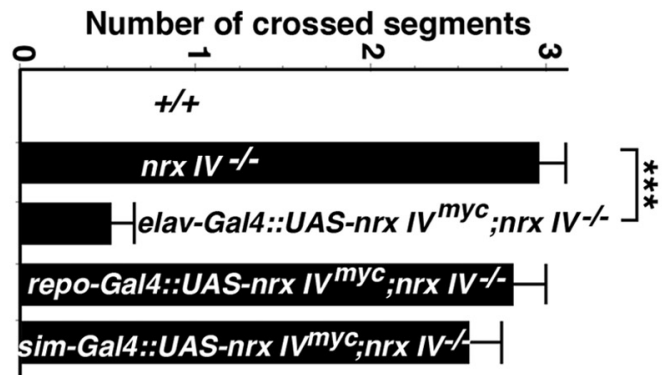

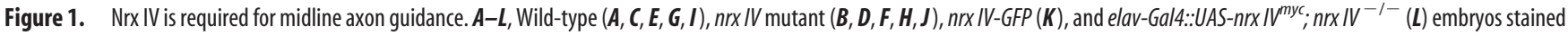
with Nrx IV, Fas II, and BP102 (A-J, $\boldsymbol{L}$ ), and GFP, Fas II, and BP102 (K). All panels show ventral views with anterior up and the optical focus is on the Fas II and BP102 axonal projections. $\boldsymbol{A}$, Stage 12 (s12) wild-type embryo shows Nrx IV localization in cell bodies of aCC ( $\boldsymbol{A} \boldsymbol{a}$, arrowhead), SP1 (arrow) and lower levels in axons. Fas II ( $\boldsymbol{A} \boldsymbol{b})$ is expressed in pCC growth cone (arrow) and BP102 (Ac) labels

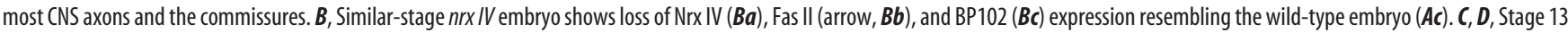
(s13) wild-type (C) and $n r x$ IV (D) embryos show localization of Nrx IV (Ca, Da), Fas II (Cb, Db), and BP102 (Cc, Dc). $\boldsymbol{E}$, Stage 14 (s14) wild-type embryo shows more refined Nrx IV localization concentrated in the boundaries between neurons and MG and lateral CNS (Ea). Fas Il expression (Eb) shows that the two axon pathways, namely, pCC/dMP2 and MP1/dMP2, are fused at the segment boundary (arrow). BP102 labels the CNS axons (EC). $\boldsymbol{F}$, Stage $14 \mathrm{nrx} / \mathrm{Vembryo}(\boldsymbol{F a})$ shows the pCC axon pathway extending anterior and toward the ML ( $\boldsymbol{F b}$, arrow). The CNS axon profiles shown with BP102 (Fc) display partially fused commissures ( $F \boldsymbol{c}$, arrow). G, Stage 15 (s15) wild-type embryo shows Nrx IV localization in neurons and axons (Ga), refinement of Fas II-labeled tracts ( $G \boldsymbol{b}$, arrow), and overall CNS axon scaffold (GC). $\boldsymbol{H}$, Stage $15 \mathrm{hrx} / V$ mutant shows increased attraction of the ipsilateral tracts toward the ML $(\boldsymbol{H b}$, arrow) and BP102 shows increasingly fused AC and PC (Hc, arrows) in most segments. I, Late-stage 16/17 (s16) wild-type embryo shows Nrx IV expression (Ia) together with Fas II that labels three distinct longitudinal axon trajectories (Ib, arrow) and BP102 (Ic).

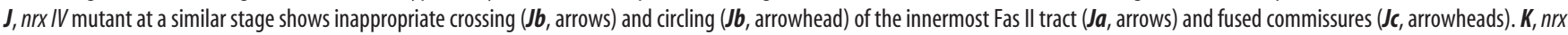
IV-GFP embryo shows distinct axonal localization of GFP $(\boldsymbol{K a})$ in addition to Fas II $(\boldsymbol{K} \boldsymbol{b})$ and BP102 $(\boldsymbol{K} \boldsymbol{c})$. $\boldsymbol{L}$, An elav-Gal4::UAS-nrx/V;nrx/V ${ }^{-1-}$ embryo shows expression of Nrx IV ( $\left.\mathbf{L a}\right)$ in all elav-Gal4-positive cells and rescue of Fas II ( $(\boldsymbol{b} \boldsymbol{b}$, arrows)- and BP102 ( $\boldsymbol{C} \boldsymbol{c}$ )-labeled axons in two of the displayed segments ( $\boldsymbol{L} \boldsymbol{d})$. $\boldsymbol{M}$, Quantification of axon crossing from 400 abdominal segments of wild-type, $n r x I V$ and elav-Gal4::UAS-nrx $I V^{m y c} ; n r x / V^{-1-}$, repo-Gal4::UAS-nrx $I V^{m y c} ; n r x I^{-1-}$, and sim-Gal4::UAS-nrx $I V^{m y c} ; n r x I V^{-1-}$ mutant embryos. Statistically significant rescue of axon crossing defects is observed only in elav-Gal4:UUAS-nrx IV ${ }^{\text {myc}} ; n r x / V^{-1-}$ embryos $(p<0.001)$ compared with $n r x$ IV mutants.

two sibling neurons, anterior corner cell (aCC) and posterior corner cell (pCC). Nrx IV localization is strong in the aCC (Fig. $1 A a$, arrowhead), pCC, and the SP1 pioneer neurons (Fig. $1 A a$, arrow). Nrx IV is also localized to the two ML precursor (MP) 1 and MP2 pioneer neurons as revealed by staining with Odd skipped and 22c10 antibodies (data not shown). At subsequent stages $13,14,15$, and late 16/17 (Fig. $1 C a, E a, G a$, Ia, respectively), Nrx IV neuronal expression becomes more refined with distinct localization in the ML. Nrx IV is uniformly distributed through- out the lateral CNS neurons and is expressed at lower levels in the axons (Fig. 1 Ia). The axonal expression of Nrx IV with nrx IV: GFP exon-trap line shows prominent GFP expression in axons of both commissures and longitudinal connectives (LCs) (Fig. $1 \mathrm{Ka}$, arrows), indicating that Nrx IV is expressed in the CNS axons.

Having established the wild-type localization of Nrx IV in the developing CNS, we next examined the consequences of loss of Nrx IV in the CNS with Fas II and BP102 antibodies as a phenotypic readout. As expected, $n r x I V$ mutant embryos display com- 
plete loss of Nrx IV protein (Fig. $1 \mathrm{Ba}, \mathrm{Da}, \mathrm{Fa}, \mathrm{Ha}, \mathrm{Ja}$ ) (Banerjee et al., 2008). At stage 12, Fas II is expressed in aCC and pCC neurons (Fig. $1 \mathrm{Ab}$ ) and in the anterior extending growth cone of pCC (Fig. $1 A b$, arrow). The commissures at this stage are clearly established but appear fused at the ML (Fig. $1 A c$ ). In a similar-stage $n r x I V$ mutant embryo, the Fas II and BP102 staining is comparable to the wild-type embryo (Fig. $1 B b-B d$ ). A stage 13 wild-type embryo (Fig. $1 \mathrm{Cb}$ ) expresses Fas II in additional axons, including MP1, dMP2, and vMP2, all of which fasciculate in the MP1 pathway. BP102 staining reveals further separations of AC and PC as the middle MG becomes intercalated between the two commissures (Fig. 1Cc) (Klämbt et al., 1991). In stage 13 nrx IV mutant embryos, no significant differences in the Fas II-expressing axons are noted (Fig. $1 \mathrm{Db}$ ) but the BP102 pattern in $n r x I V$ mutant embryo shows that commissural separation is not occurring normally (Fig. $1 D c$ ). Stage 14 wild-type embryos express Fas II in the pCC pathway, which is continuous from segment to segment (Fig. $1 \mathrm{~Eb}$, arrow) along the entire length of the CNS. The AC and PC show greater separation as revealed by BP102 staining at this stage (Fig. $1 E c$ ). At stage $14 n r x I V$ mutants begin to exhibit defects in Fas II localization. The pCC pathway becomes attracted toward the ML (Fig. $1 F b$, arrow) and commissure separation is further affected (Fig. $1 F \mathcal{C}$, arrow). The axonal phenotypes as revealed by both Fas II and BP102 become more pronounced as the $n r x I V$ mutant embryos develop. At stage 15, wild-type embryos show refinement of the ipsilateral Fas II tracts (Fig. $1 G b$, arrow), while $n r x I V$ mutant embryos show crossing of the most medial Fas II-positive pCC trajectory (Fig. $1 \mathrm{Hb}$, arrow) and thicker commissures (Fig. $1 \mathrm{Hc}$, arrow) compared with the wild-type embryos (Fig. 1Gc). Embryonic stage 16 marks the completion of entire axon scaffold formation in the CNS. By late stage, i.e., stage 16/17, wild-type embryos show Fas II expression at high levels on three longitudinal axon bundles on either side of ML (Fig. $1 \mathrm{Ib}$, arrows). BP102, on the other hand, labels all CNS axons, including the commissural and longitudinal pathways. $n r x I V$ mutants at stage 16 and beyond show the most striking axonal phenotypes, which include crossing (arrows) and often circling of Fas II-positive ipsilateral axons in the ML (Fig. $1 \mathrm{Jb}$, arrowhead) and thinning of LCs together with the failure of the AC and PC to separate, resulting in partially to completely fused commissures (Fig. $1 \mathrm{Jc}$, arrowheads). These phenotypes are similar to those observed in robo mutants (Kidd et al., 1998). To demonstrate that the axon guidance defects observed in $n r x I V$ mutant CNS are due to loss of $n r x$ $I V$ in neurons, we used the pan-neural elav-Gal4 line to drive $U A S-n r x I V^{m y c}$ in $n r x I V$ mutant background. Stage 16 embryos mutant for $n r x I V$ and carrying elav-Gal4::UAS-nrx $I V^{m y c}$ analyzed using Nrx IV (Fig. 1 La), Fas II (Fig. 1 Lb), and BP102 (Fig. $1 L c$ ) antibodies showed rescue of the axonal phenotypes (Fig. $1 L d, M)$, indicating that loss of Nrx IV from the CNS neurons results in axon guidance defects. Expression of $\mathrm{Nrx}_{\mathrm{IV}}{ }^{\mathrm{myc}}$ either in all ML cells (glia and neurons) with sim-Gal4 or in all lateral glia with repo-Gal4 failed to rescue the axon crossing phenotypes in $n r x I V$ mutants, suggesting that these CNS cell types do not contribute to the axon guidance phenotypes observed in $n r x I V$ mutants (Fig. 1M).

We further show that absence of Nrx IV results in loss of ML repulsion by studying the Apterous (Ap)-positive neurons and their ipsilaterally projecting axons in the CNS in $n r x I V$ mutants (Lundgren et al., 1995) (supplemental Fig. S1, available at www. jneurosci.org as supplemental material). We find that in $n r x I V$ mutants, the Ap neurons extend axons that display pathfinding errors, including misrouting across the ML in most of the segments (supplemental Fig. S1, available at www.jneurosci.org as supplemental material). Together, these results demonstrate that loss of Nrx IV results in loss of axonal repulsion at the ML.

\section{Nrx IV function in axon guidance is independent of Wrapper, Contactin, and Neuroglian}

Our earlier studies have shown that Nrx IV interacts with Ig superfamily proteins such as Cont, Nrg, and Wrapper (FaivreSarrailh et al., 2004; Banerjee et al., 2006; Wheeler et al., 2009). Nrx IV, Cont, and Nrg are required for the formation and maintenance of epithelial and axo-glial SJs (Faivre-Sarrailh et al., 2004; Banerjee et al., 2006). Nrx IV and Wrapper function in ensheathment of commissural axons in the CNS and in the maintenance of the midline neuron-glial scaffold (Wheeler et al., 2009). We wanted to test whether any of these interacting partners of Nrx IV displayed axon guidance phenotypes as observed in $n r x I V$ mutants. cont and $n r g$ mutants did not display axon crossing defects when stained with anti-Fas II (supplemental Fig. S2, available at www.jneurosci.org as supplemental material). Immunostaining of the wild-type embryos from stage 12 through 17 (Fig. 2A-F) show localization of Nrx IV (green) and Wrapper (red) in the developing CNS midline, in which Nrx IV in the midline neurons localizes asymmetrically in juxtaposition to Wrapper in MG (Fig. $2 A-F$, arrowheads) (Stork et al., 2009; Wheeler et al., 2009). In addition, Nrx IV expression is also observed in the lateral CNS neurons and axons (Fig. $2 A c, E c$, arrows). To determine whether wrapper mutants displayed axon guidance defects, wild-type (Fig. $2 G$ ) and wrapper mutant (Fig. $2 H$ ) embryos were triple immunostained with Wrapper, Fas II, and BP102 antibodies. The wildtype embryos show normal localization of Wrapper in MG (Fig. $2 G a$, green), ipsilateral projections of Fas II-positive axons (Fig. $2 G b$, red) and BP102 labeling of the CNS axons (Fig. 2Gc, blue) and together in the merged image (Fig. $2 G d$ ). Similar-stage wrapper mutant embryos (Fig. $2 H$ ) do not display any significant axon guidance defects when labeled with anti-Fas II (Fig. $2 \mathrm{Hb}$, red) and BP102 (Fig. $2 \mathrm{Hc}$, blue). These studies reveal that the axon guidance functions of Nrx IV are independent of its known binding partners Cont, Nrg, and Wrapper.

\section{Robo is diffusely distributed in longitudinal and commissural axons of $n r x I V$ mutants}

Since $n r x I V$ mutants display crossing and circling of ipsilateral axons together with collapse of commissural axons at the ML, and because the axon guidance function of Nrx IV is independent of its binding partners Wrapper (Fig. 2), Contactin, and Neuroglian (supplemental Fig. S2, available at www.jneurosci.org as supplemental material) (Noordermeer et al., 1998; FaivreSarrailh et al., 2004), we reasoned that Nrx IV might partner with other proteins for its axon guidance function in the ML. Axon guidance in the ML can be regulated both by attractive and repulsive cues (Garbe and Bashaw, 2007; Yang et al., 2009). We examined the localization of Nrx IV in netrin and frazzled mutants (supplemental Fig. S3, available at www.jneurosci.org as supplemental material) and did not find any striking anomaly in Nrx IV localization or a similarity in its axonal guidance phenotypes. Given that $n r x I V$ axon guidance phenotypes resemble that of the robo mutants (Kidd et al., 1998), we hypothesized that Nrx IV most likely functions in the Slit/Robo repulsive signaling pathway (Brose et al., 1999; Kidd et al., 1999; Fan et al., 2003). Therefore, we first examined the localization patterns of Nrx IV and Robo in the wild-type embryo and nrx IV and robo mutants (Fig. 3 ) to determine whether these two proteins are interdependent for their localization in the CNS. 

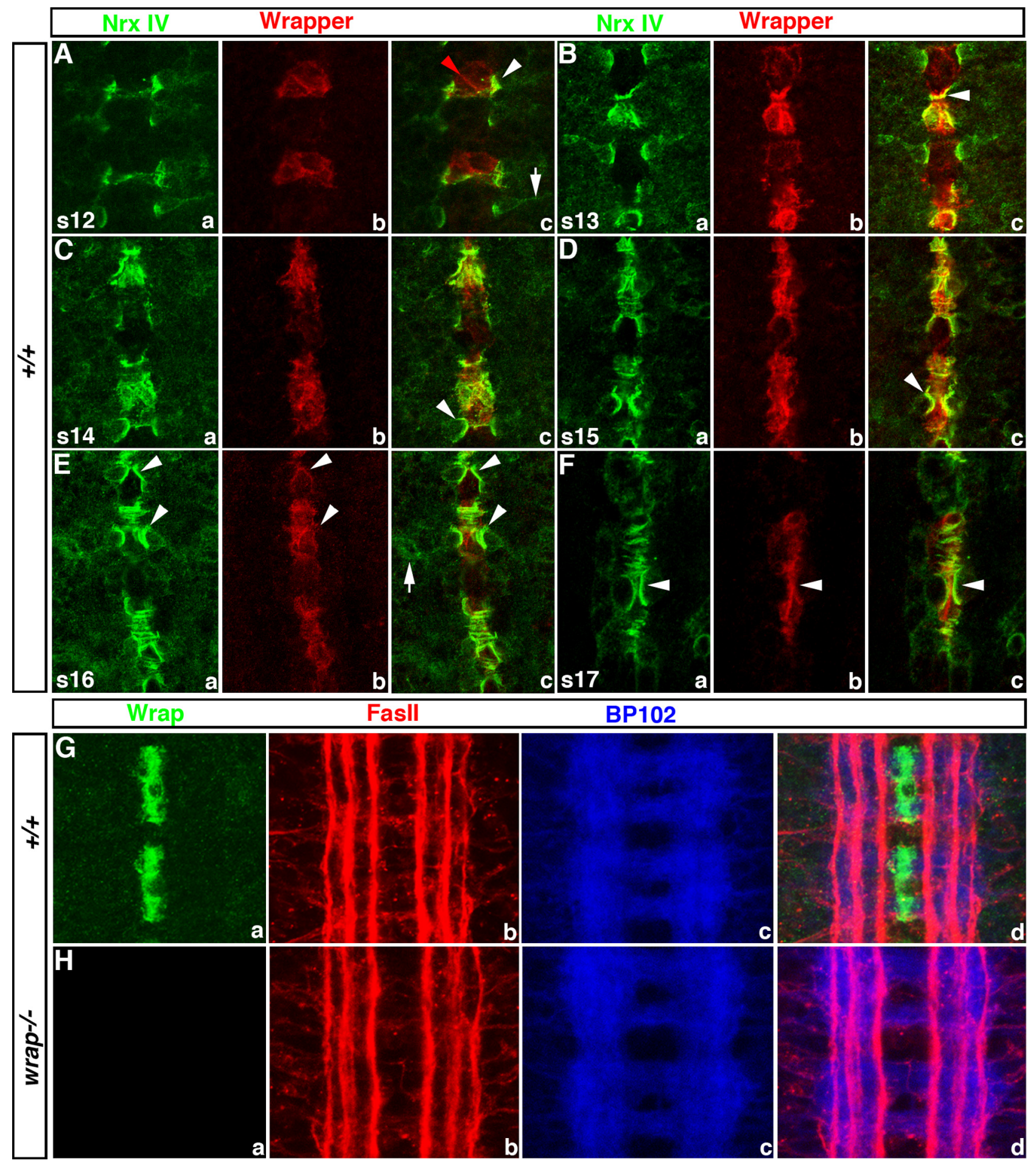

Figure 2. Developmental expression and localization of Nrx IV and Wrapper in the CNS midline and axon guidance in wrapper mutants. All panels shown are the ventral view with anterior up. In panels $\boldsymbol{A}-\boldsymbol{F}$, the optical focus is at the MG-neuron interface. $\boldsymbol{A}-\boldsymbol{F}$, Wild-type embryos of stages $12(\boldsymbol{A}), 13(\boldsymbol{B}), 14(\boldsymbol{C}), 15(\boldsymbol{D}), 16(\boldsymbol{E})$, and $17(\boldsymbol{F})$ showing localization of Nrx IV (green, $\boldsymbol{A a}, \boldsymbol{B} \boldsymbol{a}$, $\boldsymbol{C a}, \boldsymbol{D a}$, $\mathbf{E} \boldsymbol{a}, \boldsymbol{F} \boldsymbol{a}$ ) and Wrapper (red, $\boldsymbol{A} \boldsymbol{b}, \boldsymbol{B} \boldsymbol{b}, \boldsymbol{C} \boldsymbol{b}, \boldsymbol{D b}, \boldsymbol{E} \boldsymbol{b}, \boldsymbol{F} \boldsymbol{b}$ ) in the developing $\mathbf{C N S}$ midline. Nrx IV shows a strong asymmetric localization (white arrowheads) in juxtaposition to Wrapper localization in the MG $(A c, B c, D c, E c, F c)$. Arrows in $A c$ and $E c$ show expression of Nrx IV in axons and lateral CNS neurons. Red arrowhead in $A c$ indicates Wrapper localization at MG interface, whereas Nrx IV and Wrapper are at the MG/ML neuron interface (Wheeler et al., 2009). G, Stage 16 wild-type embryos show Wrapper localization in MG (Ga) and axonal profile with anti-Fas II (Gb) and BP102 (Gc) and in merged image (Gd). $\boldsymbol{H}$, Stage 16 wrapper mutants $(\boldsymbol{H a})$ shows no detectable Wrapper protein as expected $(\boldsymbol{H a})$ and display normal trajectories of axonal profiles with no noticeable axon guidance defects, as is evident from staining with anti-Fas II $(\boldsymbol{H b})$ and BP102 $(\boldsymbol{H c})$ and in merged image $(\boldsymbol{H d})$.

Robo expression in stage 12 wild-type embryos is seen on the growth cones, which project ipsilaterally (Kidd et al., 1998). Little or no Robo is observed on commissural axons as they extend toward and across the ML. However, earlier work has shown that as the commissural growth cones turn to project longitudinally, their level of Robo expression dramatically increases (Kidd et al., 1998). During stages 14-16 of embryonic development, Robo continues to be expressed at high levels on all longitudinally pro- 

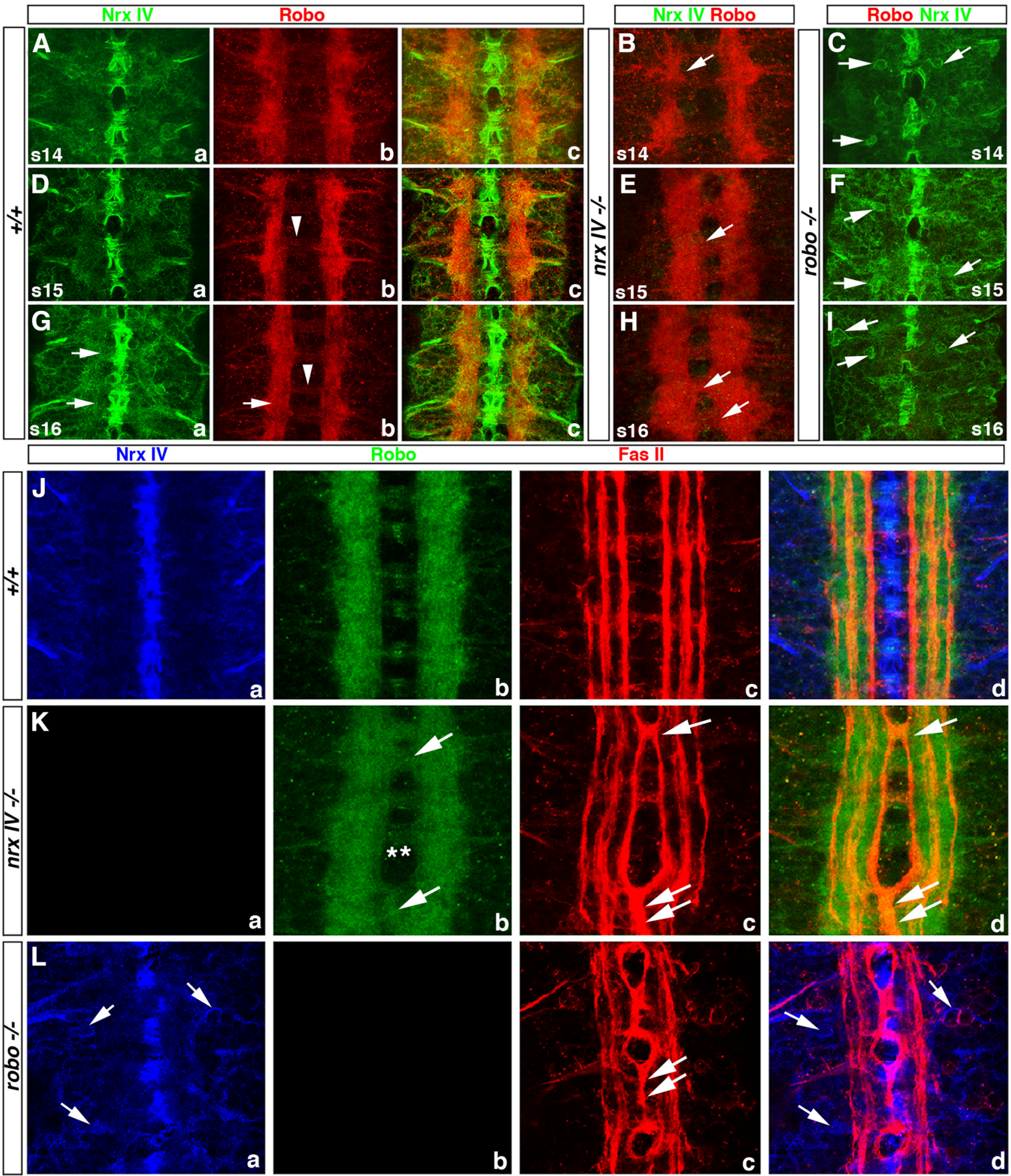

Figure 3. Localization of Nrx IV and Robo in $n r x / V$ and robo mutants. All panels show ventral views with anterior up. $\boldsymbol{A}-\boldsymbol{I}$, Stage 14 wild-type embryo with Nrx IV ( $\boldsymbol{A} \boldsymbol{a})$ and Robo ( $\boldsymbol{A} \boldsymbol{B})$ expression in the CNS. Axonal staining of Nrx IV overlaps with high levels of Robo in longitudinal axons ( $\boldsymbol{A C}$ ). $\boldsymbol{B}, \boldsymbol{C}$, Increased Robo localization is seen on contralateral axon tracts in stage $14 \mathrm{nrx} / \mathrm{V}$ mutants ( $\boldsymbol{B}$, arrow), while Nrx IV localization in robo mutant is mildly affected and localizes more in lateral CNS neuronal cell bodies (C, arrows). $\boldsymbol{D}$, Stage 15 wild-type embryo shows Nrx IV (Da) and Robo (Db) expression with Robo at a higher level in axons, while a lower level is detected in the commissures (Db, arrowhead). $E$, Stage 15 nrx /V embryos show uniform Robo distribution in commissural and longitudinal axons (arrow). $\boldsymbol{F}$, Nrx IV shows increased localization in cell bodies of robo mutants (arrows) compared with a similar stage in the wild-type embryo (Da). $\boldsymbol{G}$, Stage 16 wild-type embryo continues to show Nrx IV and Robo expression overlapping in the longitudinal axons $(\mathbf{G} \boldsymbol{G}, \mathbf{G} \boldsymbol{b}$, arrows). Arrowhead in $\mathbf{G} \boldsymbol{b}$ points to low level of Robo detected in the commissures. $\boldsymbol{H}$, Stage $16 \mathrm{nrx} / V$ embryos show uniformly diffuse distribution of Robo in the longitudinal and commissural axons (arrows). I, Nrx IV localization appears to be increased in cell bodies in robo mutants at a similar stage

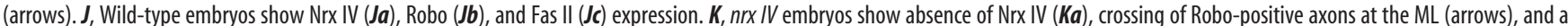
uniformly diffused localization of Robo in both the longitudinal and the commissural tracts ( $\boldsymbol{K} \boldsymbol{b}$, asterisks). Fas II-labeled axons cross at multiple segments in the ML (Kc, arrows), and display a complete fusion of both of the innermost tracts (Kc, double arrows). $\boldsymbol{L}$, Nrx IV shows increased distribution in neuronal cell bodies (arrows) in robo mutants (La) compared with a similar stage in the wild-type embryo (Ja). Fas II labeling shows multiple crossing and circling of axons and fused innermost tracts ( $\mathbf{L}$, double arrows) similar to $\mathrm{nrx} / \mathrm{V}$ mutants (Kc). 
jecting growth cones and axons and at low levels on commissural axons (Fig. $3 A b, D b, G b$, respectively). Nrx IV localization in stages 14-16 (Fig. 3Aa,Da, Ga) is as described in Figure 1. Robo and Nrx IV expression overlaps in the longitudinal axons of the lateral CNS as seen in the merged image (Fig. $3 A c, D c, G c$ ). Robo localization in $n r x I V$ mutants or vice versa at embryonic stages 12 and 13 resembles the wild-type localization (data not shown). Starting around stage 14, nrx IV mutants display Robo localization in crossing axons (Fig. 3B, arrow). At stages 15 and 16, nrx IV mutants show diffuse Robo distribution in commissural and longitudinal axon tracts (Fig. $3 E, H$, arrows, $K b$ ). Similarly, in robo mutants at stages 14 through 16, Nrx IV displays aberrant distribution in both the ML and lateral CNS and is observed more in neuronal cell bodies (Fig. 3C,F,I, arrows) compared with the wild-type embryos at similar stages of development (Fig. $3 A a, D a, G a)$. These data indicate that Nrx IV and Robo are dependent on each other for their proper localization in the CNS.

To further investigate the Robo localization with respect to misguided Fas II-positive axons in $n r x I V$ mutants, we studied the coexpression of Robo and Fas II (Fig. 3J-L). Robo localization in the wild-type embryos (Fig. $3 \mathrm{Jb}$ ) is at high levels on the longitudinal tracts on either side of the ML. Fas II-positive axons (Fig. $3 J c$ ) show normal ipsilateral axon trajectories in the wild-type embryos. In $n r x I V$ mutants, however, Fas II-labeled axons cross the ML at several segments (Fig. $3 \mathrm{Kc}$, arrows) and often display a complete collapse of the two innermost axon tracts at the ML as is seen in robo mutants (Fig. $3 K c, L c$, double arrows). Interestingly, the distribution of Robo in $n r x I V$ mutants appears diffuse and uniform in both the longitudinal and commissural tracts (Fig. $3 \mathrm{~Kb}$, arrows and asterisks). We further noticed two aspects of Robo phenotype in $n r x I V$ mutants with respect to Fas II. First, Robo-positive tracts in few segments cross and seem to collapse at the ML, in which Fas II-labeled axons do not cross. Second, in the majority of other segments in which the Fas II ipsilateral axons cross the ML, Robo-positive axons also show increased crossing and a more pronounced collapse at the ML. These studies further confirm that Robo localization is dependent on Nrx IV as evidenced by the uniformly diffuse distribution of Robo in longitudinal tracts and commissures regardless of whether Fas II axons cross or not. In robo mutants Nrx IV localization is also altered and more Nrx IV is observed in neuronal cell bodies (Fig. 3La,d, arrows).

\section{$n r x I V$ exhibits dose-dependent genetic interactions with robo}

Dosage-sensitive genetic interactions between two loci are a good indicator that the proteins are functionally related. We therefore tested for genetic interactions between robo and $n r x I V$. We examined the CNS of embryos transheterozygous or homozygous for $n r x I V$ and robo by generating robo ${ }^{G A 285} / C y O ; n r x I V^{4304} / T M 6$ flies. The wild-type embryos, single mutants, and combinations of robo and $n r x I V$ mutants were immunostained against Nrx IV and Robo to identify mutant genotypes. Fas II (Fig. 4A-G) and BP102 (Fig. $4 H-N$ ) served as the phenotypic readout of the axonal defects at the ML in stage 16 embryos. In wild-type embryo, Fas II labels three parallel axon fascicles that do not cross the ML (Fig. 4A). nrx IV mutants show crossing of the innermost Fas II trajectory (Fig. $4 B$, arrow), often resembling the robo mutants (Fig. $4 C$, arrow). robo/+;nrx IV/+ transheterozygotes also display ML crossing of Fas II that is significantly higher than that of either $n r x$ $I V /+$ or robo/+ heterozygotes (Fig. $4 \mathrm{D}$, arrow, $\mathrm{O}$ ). This dosagedependent transheterozygous phenotype is a strong indication that Nrx IV and Robo interact directly and function in the same pathway. The Fas II phenotype of homozygous nrx IV mutants is significantly enhanced by removing one copy of robo (Fig. $4 E$, arrows) while loss of one copy of $n r x I V$ from robo homozygous mutants (Fig. $4 F$ ) does not significantly enhance the robo mutant phenotype (compare with Fig. $4 C, O$ ). Double mutant robo ${ }^{-1-}$; $n r x I V^{-l-}$ embryos showed phenotypes that resembled robo mutant (Fig. 4G). Some robo ${ }^{-1-} ; n r x I V^{-1-}$ embryos occasionally displayed more severe defects but the severity of defects in Fas II tracts was not statistically significant compared with robo single mutants (compare with Fig. 4C,O). Quantitative analyses of the Fas II phenotypes (Fig. 4A-G) clearly highlight a dose-dependent genetic interaction between the two loci (Fig. 4O).

We next studied the overall axon profile in the CNS of embryos from each of the above genotypes (Fig. $4 \mathrm{H}-\mathrm{N}$ ) by staining with $\mathrm{BP} 102$. In wild-type embryos the BP102-labeled axon scaffold exhibits a characteristic ladder-like appearance (Fig. 4H). nrx IV mutants show partial to complete fusion of commissures in most segments and comparatively thinner LCs (Fig. 4I, arrows). robo mutants show a severe disruption of the CNS axon scaffold with thicker AC and PC and thinner LCs (Fig. 4J, arrows) (Seeger et al., 1993; Kidd et al., 1998). We next analyzed axonal phenotypes in embryos heterozygous for robo and $n r x I V$ (Fig. $4 K$ ); embryos lacking one copy of robo and two copies of $n r x I V$ (Fig. $4 L$ ); embryos missing two copies of robo and one copy of $n r x I V$ (Fig. $4 M$ ); and embryos double mutant for robo and $n r x I V$ (Fig. $4 N$ ). As seen in Figure $4 K$, transheterozygotes of robo and $n r x I V$ show $4-5 \%$ of segments with a mild reduction in the intercommissural distance and reduced spacing between the LCs compared with the wild-type embryos (Fig. $4 \mathrm{H}$ ). The effect of removing one copy of robo in nrx IV mutants resulted in a dominant enhancement of BP102 phenotype compared with $n r x I V$ mutants alone (Fig. 4, compare $L$ with $I$ ). However, removal of one copy of $n r x I V$ from robo mutant embryos did not cause significant aggravation of the BP102 phenotype (Fig. 4M) compared with robo-null mutants (Fig. $4 J$ ). robo, nrx IV double mutant embryos displayed a collapse of commissures (Fig. $4 \mathrm{~N}$ ). These data indicate a range of axonal phenotypes that includes significantly thicker commissures, reduced to sometimes missing parts of LC in various genotypes, strongly indicating a loss of ML repulsion. Together, these studies demonstrate dosage-sensitive genetic interactions between nrx IV and robo, providing evidence that they function together in repulsive axon guidance pathway.

We also studied Robo localization with respect to other ipsilateral trajectories such as Ap-positive axons. Robo (Fig. $4 \mathrm{~Pa}$ ) and Ap-positive axons labeled by GFP (Fig. $4 P b$ ) in ap-Gal4::UAS-tau-GFP embryo show Robo localization as described in Figure 3, while Ap axons show normal ipsilateral axon trajectories. In ap-Gal4::UAS-tau-GFP;nrx $I V^{-1-}$ embryos Robo show diffuse localization in LCs and commissures (Fig. 4Qa), while the GFP-labeled Ap axons also cross the ML (Fig. 4Qb, arrows), further suggesting that different classes of ipsilateral axons are influenced by loss of ML repulsion in $n r x I V$ mutants.

nrx IV exhibits dose-dependent genetic interactions with slit The well established role of Slit as a ligand for Robo receptors in repulsive axon guidance led us to hypothesize that Nrx IV might also interact with Slit. We thus determined whether or not Nrx IV, Slit, and Robo are interdependent for their proper localization and/or function. Immunofluorescent labeling of stage 16 wild-type embryos with Nrx IV (Fig. 5Aa), Slit (Fig. 5Ab), and Robo (Fig. $5 A c$ ) shows that Nrx IV protein is concentrated at the boundaries between the MG and the neuronal soma and is also expressed in the lateral CNS (Fig. 5Aa,Ad) (Wheeler et al., 2009), 


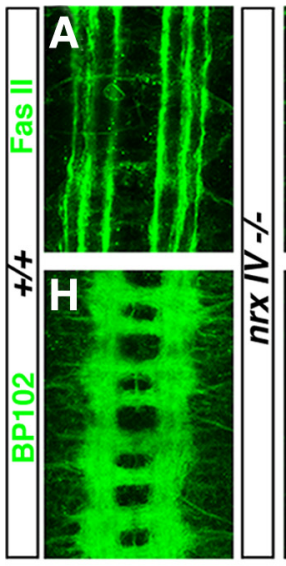

O
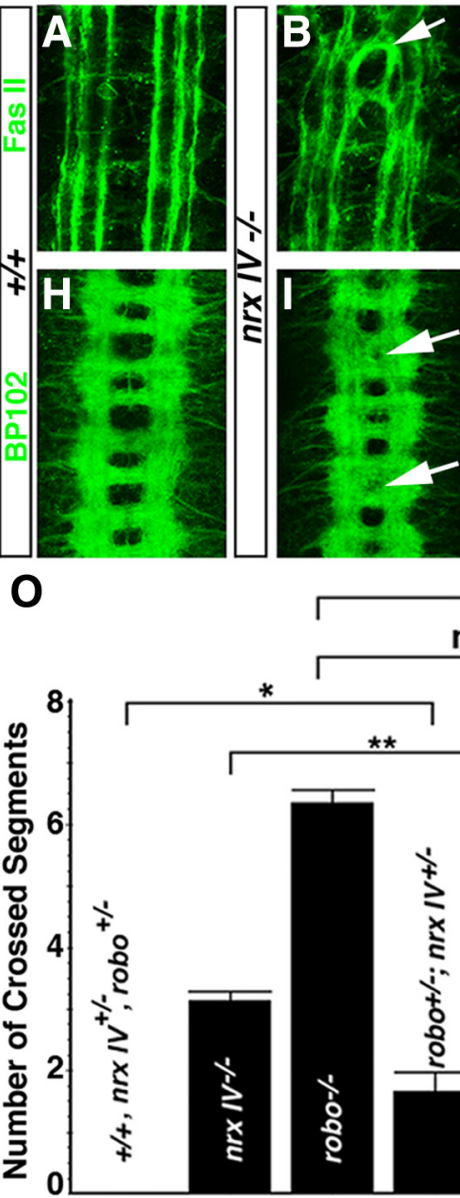

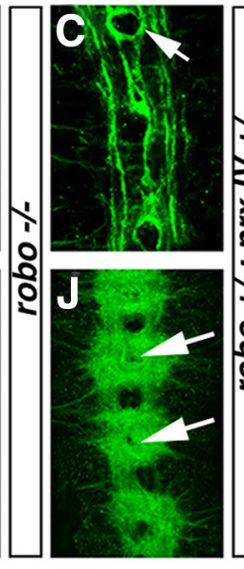

ns
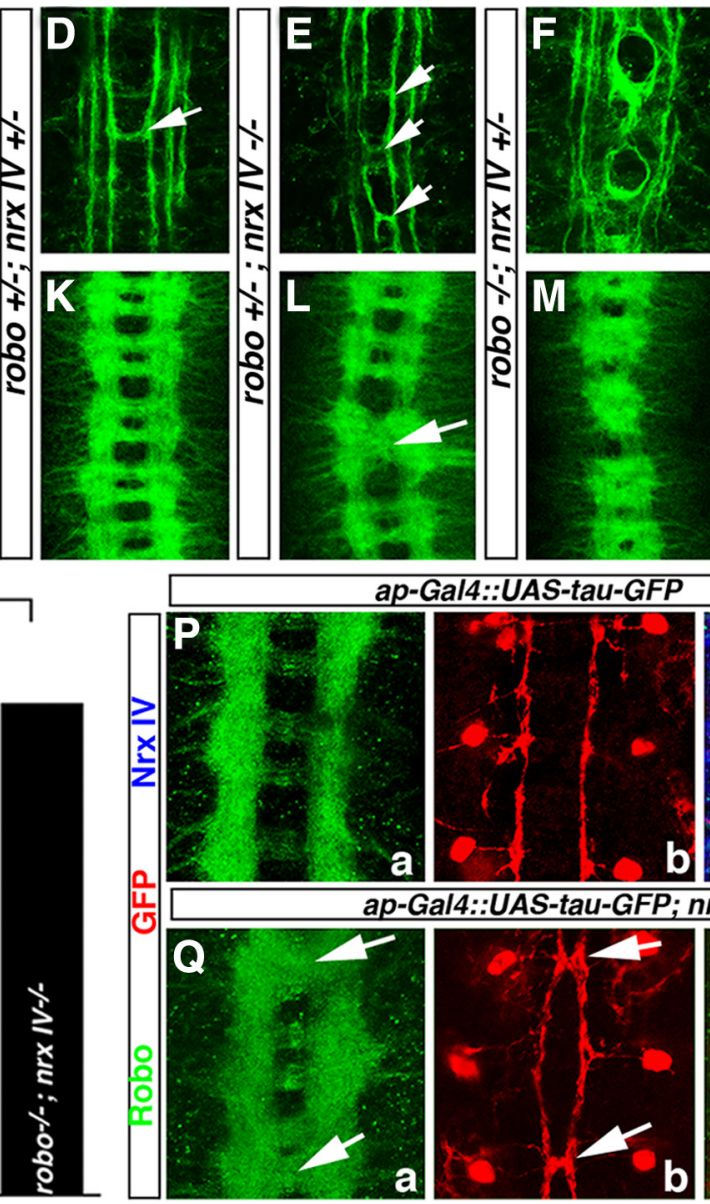
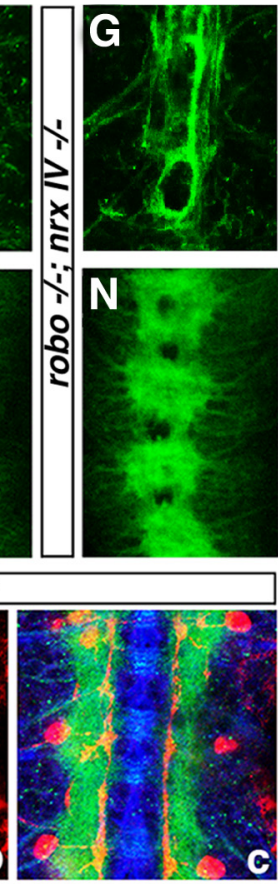

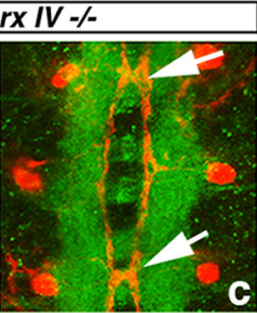

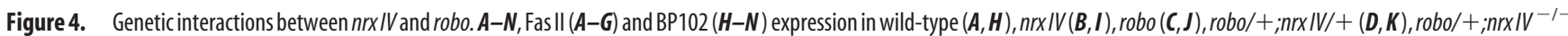
$(\boldsymbol{E}, \boldsymbol{L})$, robo $0^{-I-} ; n r x I V /+(\boldsymbol{F}, \boldsymbol{M})$, and robo ${ }^{-I-} ; n r x I^{-I-}(\boldsymbol{G}, \boldsymbol{N})$ embryos. Arrows indicate crossing axons $(\boldsymbol{B}-\boldsymbol{E})$ and fused commissures $(\boldsymbol{I}, \boldsymbol{J}, \boldsymbol{L})$. $\mathbf{O}, \mathbf{Q}$ uantitative analyses of FAS $\|$ axons crossing the midline in various genotypic combinations $(\boldsymbol{A}-\mathbf{G})$. The asterisk(s) denotes the significance between the genotypes in comparison. $\boldsymbol{P}, \mathbf{Q}$, ap-Gal4::UAS-tauGFP $(\boldsymbol{P})$ and ap-Gal4::UAS-tauGFP; $n$ rx $I^{-I-}(\mathbf{Q})$ embryos expressing Robo $(\mathbf{P a}, \mathbf{Q} \boldsymbol{a}), \operatorname{GFP}(\mathbf{P b}, \mathbf{Q} \boldsymbol{b})$, and $\operatorname{Nrx} \operatorname{IV}(\mathbf{P} \boldsymbol{c}, \mathbf{Q} \boldsymbol{c}$, blue $)$.

while Slit localizes mostly in the MG and appears diffuse in the commissural tracts (Fig. 5Ab,Ad) (Kidd et al., 1999). Robo localization is as described in Figure 3. Earlier studies have shown that Slit protein is synthesized in the MG and is eventually associated with the surfaces of axons that traverse them (Rothberg et al., 1990; Kidd et al., 1999). In $n r x I V$ mutants, Slit is present in the MG but Slit levels are qualitatively reduced (Fig. $5 B$ ), while in slit mutants, the localization of Nrx IV in the ML is severely altered (Fig. 5Ca, arrows) and Robo-positive axons show complete collapse at the ML (Fig. 5Cc, arrowheads) (Kidd et al., 1999). In robo mutants, Slit levels in MG are reduced and show a punctate distribution and aberrant localization compared with the wild-type embryos (Fig. 5Db, arrows). The altered localization of these proteins in the mutant backgrounds of one another could be attributed to their interdependence for proper localization or a disorganization of the MG/neuronal cytoarchitecture. Immunostaining of stage $16 \mathrm{nrx} I \mathrm{~V}$, robo, and slit mutant embryos against the MG-specific marker, Wrapper, showed alterations in the MG organization (Fig. 5U-W, respectively; compare with wild-type embryos in Fig. 5T) (Stork et al., 2009; Wheeler et al., 2009). However it should be noted that the changes in MG organization and architecture in robo and slit mutants are thought to be secondary phenotypes that are manifested during later stages of embryonic development (Kidd et al., 1999).

The genetic analysis of robo and nrx IV prompted us to also test for genetic interactions between $n r x I V$ and slit. To analyze the severity and range of the phenotypes in various transallelic combinations of slit and $n r x I V$, we generated slit ${ }^{2} / C y O ; n r x$ $I V^{4304} /$ TM6 flies and used BP102 (Fig. 5E-K) and Fas II (Fig. $5 L-R)$ as phenotypic readouts. Wild-type embryos show a ladder-like arrangement of axon trajectories in the CNS upon staining with BP102 (Fig. 5E). nrx IV mutants show thinning of LCs and fused commissures in some of the segments (Fig. $5 F$, arrow). slit mutants, on the other hand, show a complete collapse of axons at the ML (Fig. 5G). Transheterozygotes for slit and $n r x$ $I V$ display partially fused commissures (Fig. $5 H$, arrows) when compared with the wild-type embryos (Fig. $5 E$ ). There is a strong enhancement of BP102 phenotype when one copy of slit is removed from homozygous nrx IV mutants (Fig. 5I) as most segments have completely fused commissures (arrows) and thinner LCs. Embryos of slit ${ }^{-1-} ; n r x I V /+$ (Fig. 5J) and double mutants slit $^{-1-} ;$ nrx $I V^{-1-}$ (Fig. $5 \mathrm{~K}$ ) show a complete collapse of all axons in the ML similar to those of slit mutants (Fig. $5 G$ ). The BP102 phenotype in various genetic backgrounds (Fig. $5 E-K$ ) indicates that $n r x I V$ and slit display genetic interactions.

We next studied the profile of Fas II trajectories in various genotypic combinations of slit and $n r x I V$ (Fig. $5 L-R$ ). Wild-type embryos display three Fas II-positive fascicles on each side of the ML (Fig. 5L). nrx IV mutants showed abnormal crossing of the innermost Fas II-positive fascicles (Fig. 5M, arrows). Homozygous slit ${ }^{2}$ embryos showed a characteristic collapse of CNS axon scaffold onto the ML (Fig. 5N) (Kidd et al., 1999). Embryos het- 


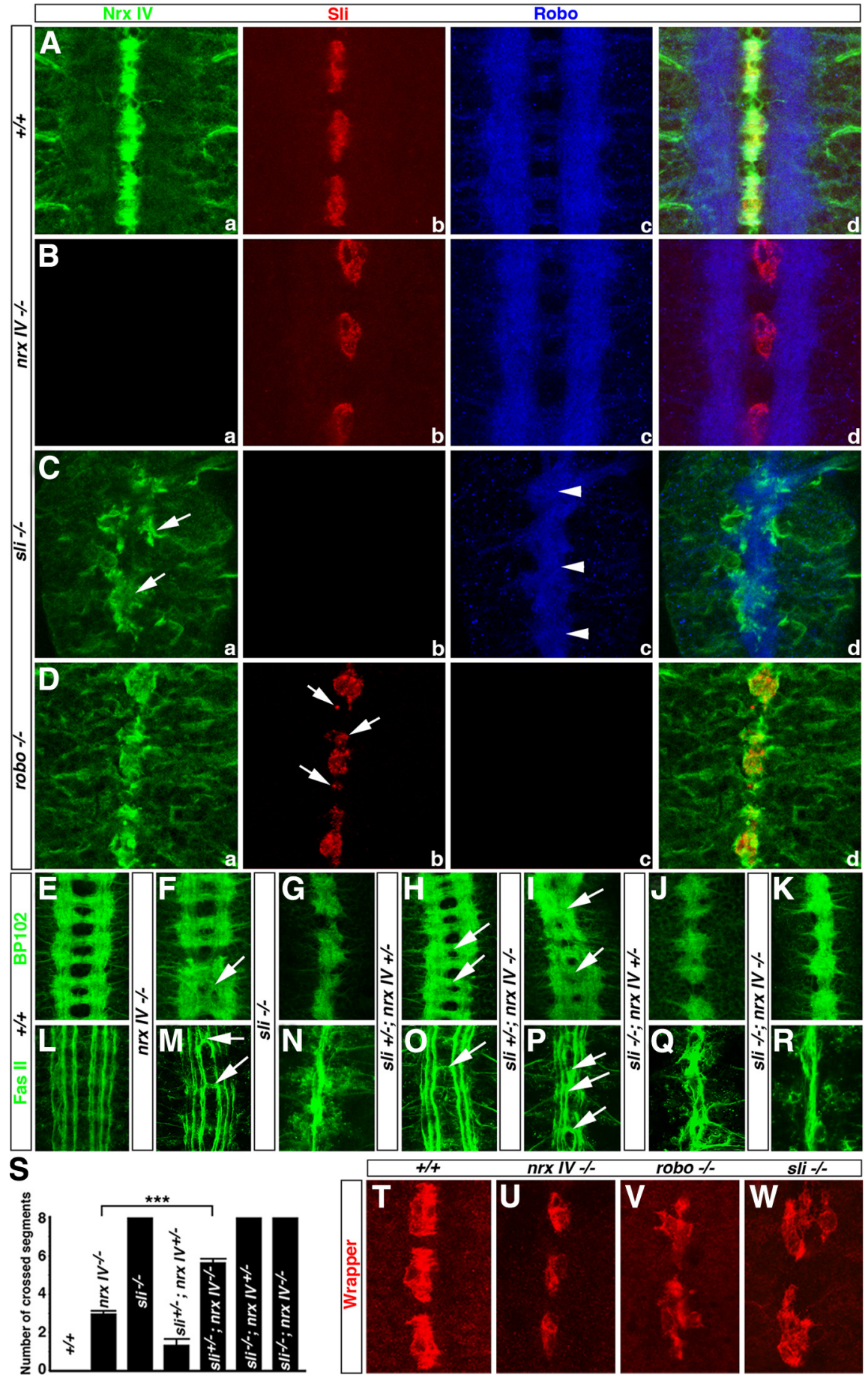

Figure 5. Subcellular localization of Nrx IV in slit mutants and genetic interactions between $n r x / V$ and slit. $\boldsymbol{A}-\boldsymbol{D}$, Wild-type $(\boldsymbol{A})$,

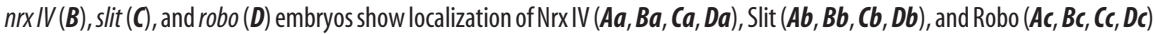
$\boldsymbol{E}-\boldsymbol{R}, \mathrm{BP} 102(\boldsymbol{E}-\boldsymbol{K})$ and Fas II $(\boldsymbol{L}-\boldsymbol{R})$ expression in wild-type $(\boldsymbol{E}, \boldsymbol{L}), n r x I V(\boldsymbol{F}, \boldsymbol{M})$, slit $(\mathbf{G}, \boldsymbol{N})$, slit/ $+; n r x I V /+(\boldsymbol{H}, \mathbf{0})$, slit ${ }^{+/-} ; n r x$ $I V^{-I-}(\boldsymbol{I}, \boldsymbol{P})$, slit ${ }^{-1-} ; n r x I V^{+--}(\boldsymbol{J}, \boldsymbol{Q})$, and slit ${ }^{-1-} ; n r x I V^{-1-}(\boldsymbol{K}, \boldsymbol{R})$ embryos. S, Quantitative analyses of the axon crossing phenotypes in $n r x I V$, slit, and various transallelic combinations $(\boldsymbol{L}-\boldsymbol{R})$. $\boldsymbol{T}-\boldsymbol{W}$, Stage 16 wild-type $(\boldsymbol{T}), n r x I V(\boldsymbol{U})$, robo $(\boldsymbol{V})$, and slit (W) embryos show Wrapper localization in MG.

erozygous for slit and $n r x I V$ showed some segments with Fas II-positive ipsilateral axons inappropriately crossing the ML (Fig. 50 , arrow). We next examined the effect of removing one copy of slit on the $n r x I V$ phenotype and found that slit dominantly enhances the nrx $I V$ phenotype as increased number of axons cross and circle the ML and the phenotype resembles that of the robo mutants (Fig. $5 P$, arrows, compare with $M$; also see Fig. $5 S$ ). Such a dosage-dependent increase in the severity of phenotype is a strong indication that Nrx IV and Slit likely function in the same pathway. It is important to note that slit heterozygotes do not display any axon guidance phenotypes (Kidd et al., 1999). Removal of one copy of $n r x I V$ does not cause any noticeable change in the already severely affected Fas II-positive tracts in slit mutants (Fig. 5Q). Similarly, double mutants of both slit and $n r x I V$ resemble the slit-null phenotype (Fig. $5 R, S$ ). Together, these data indicate that mutations in $n r x I V$ and slit show strong genetic interactions in modulating the ML repulsion phenotypes and that the subcellular localization of Slit and Nrx IV is severely altered in $n r x I V$ and slit mutants, respectively.

\section{Nrx IV, Robo, and Slit exist as a molecular complex}

To test whether the protein colocalization and genetic interactions observed between Nrx IV, Robo, and Slit reflect in vivo biochemical changes and interactions between these proteins, we performed immunoblot (IB) and co-IP analyses. We analyzed equal amounts of total proteins in embryonic extracts from identically processed COPAS-sorted wild-type and nrx IV, robo, and slit homozygous mutant embryos (see Materials and Methods) by immunoblotting to determine changes in protein levels. All mutant embryo collections were manually checked for any contaminating twi-GFP-expressing embryos (sorting efficiency close to $100 \%$ ) before they were processed for biochemical analyses. In robo mutants, Nrx IV levels were significantly reduced, and in slit mutants, Nrx IV levels were slightly increased (Fig. $6 A a)$. In $n r x I V$ and slit mutants, Robo levels were slightly reduced (Fig. 6Ab). In $n r x I V$ and robo mutants, Slit levels were unchanged or slightly reduced, respectively (Fig. 6Ac). In slit mutants, some Slit protein persists likely due to maternal contribution (Fig. 6Ac) (Furrer et al., 2007), but immunostaining of slit embryos shows no detectable Slit protein in the ML (Fig. 5Cb). Immunoblotting against $\beta$-tubulin was used as a protein loading control (Fig. 6Ad). These data indicate that loss of Robo and Slit exert opposite effects on the steady-state levels of Nrx IV, further strengthening the genetic interaction observations presented earlier.

Next we wanted to establish whether Nrx IV, Slit, and Robo form an in vivo biochemical complex. As shown in Figure $6 B$, both Robo (Fig. 6Bb) and Slit (Fig. 6Bc) are present in IP complexes with anti-Nrx IV antibodies with wild-type embryonic lysates. IP complexes from $n r x I V$ mutants with anti-Nrx IV an- 

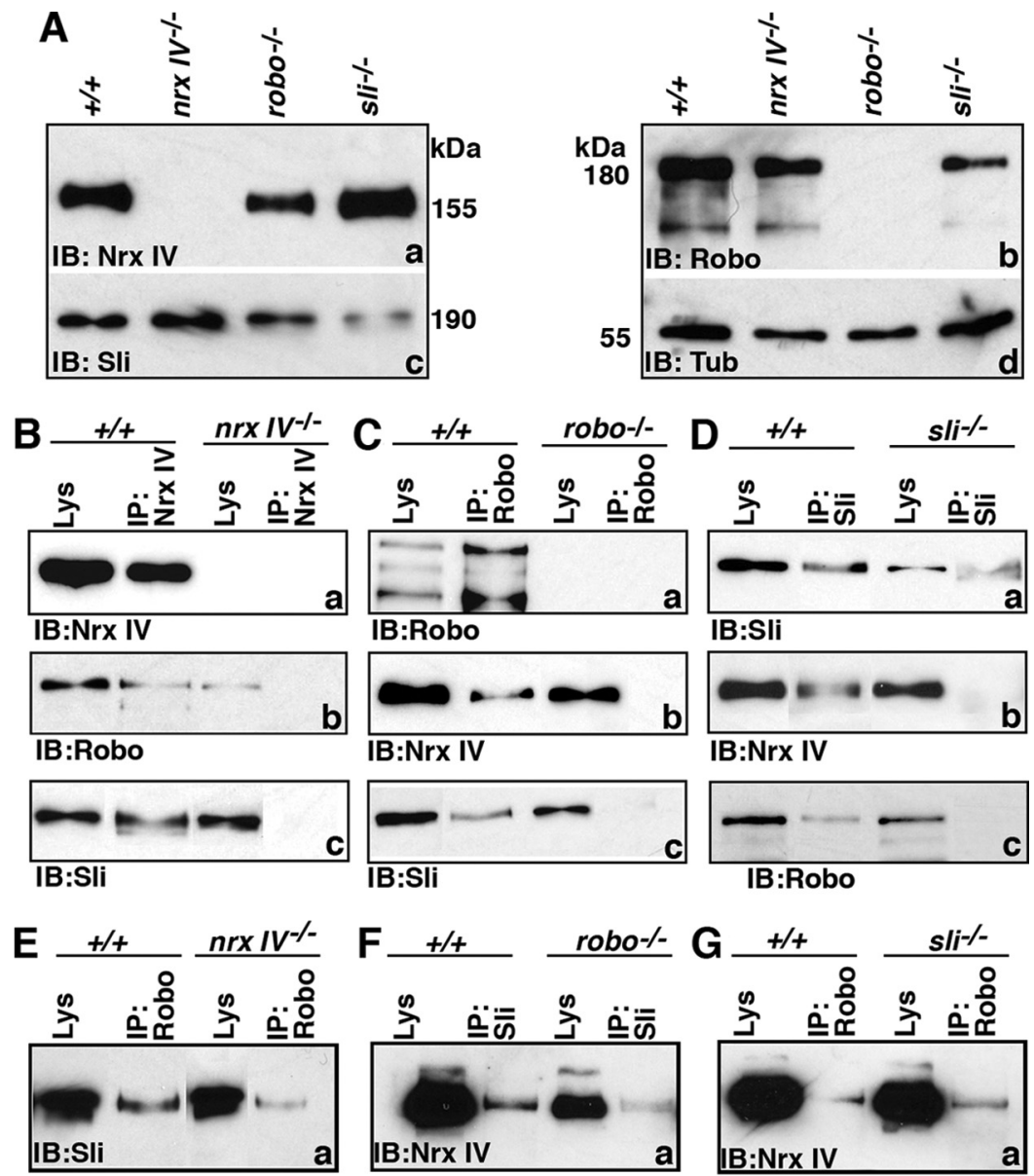

IB:Robo

a
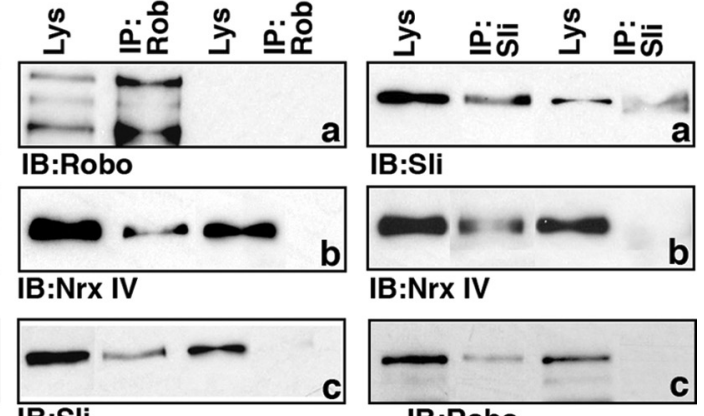

IB:Nrx IV

IB:Sli

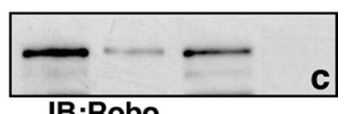

IB:Robo
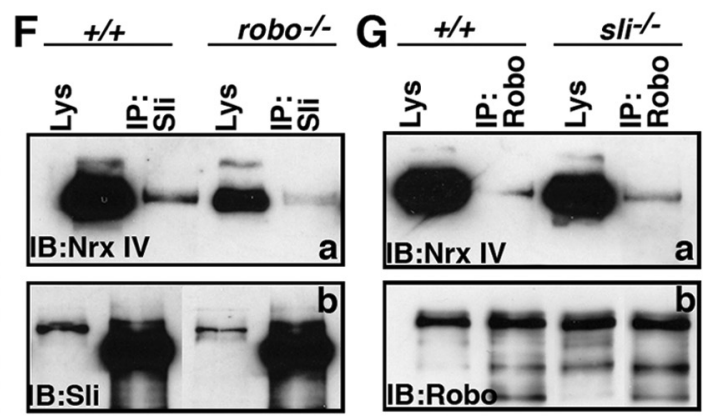

$\mathrm{H}$

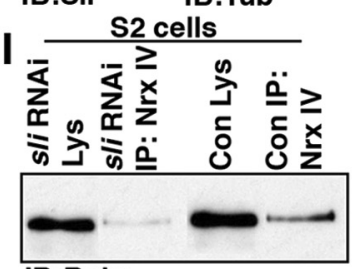

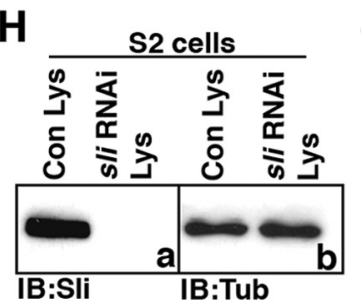

IB:Robo
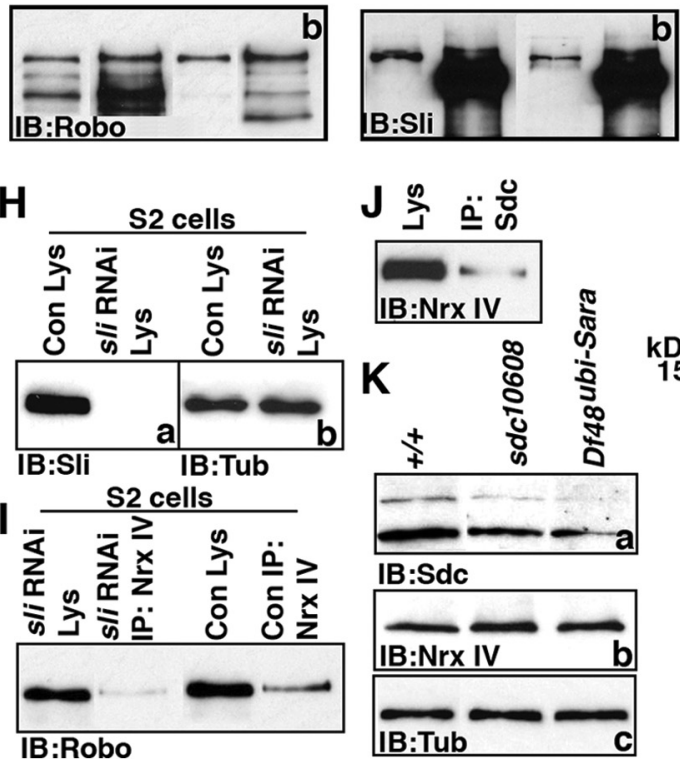

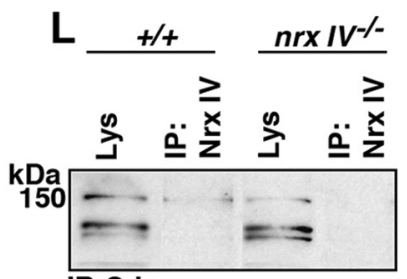

IB:Sdc

$\mathrm{M}$

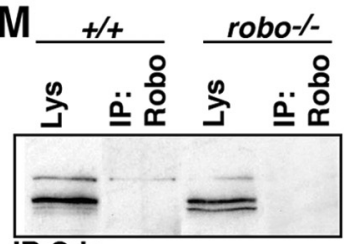

IB:Sdc

Figure 6. Nrx IV forms a biochemical complex with Robo, Slit, and Sdc. A, Western blots from equal amounts of whole embryonic lysates from wild-type and $n r x I V$, robo, and slit mutant embryos were immunoblotted for Nrx IV (155 kDa) (Aa), Robo (180 $\mathrm{kDa})(\boldsymbol{A} \boldsymbol{b})$, Slit (190 kDa) $(\boldsymbol{A c})$, and $\beta$-tubulin (55 kDa) (Ad). Nrx IV levels in robo seem to be comparatively lower than those in the wild-type and the slit mutant embryos $(\boldsymbol{A a})$. Robo levels are lower in $n r x / V$ and slit mutants $(\boldsymbol{A} \boldsymbol{b})$. Slit levels in $n r x / V$ are comparable to the wild type, while being lower in the robo mutant $(\boldsymbol{A c})$. While there is complete lack of Nrx IV and Robo from $n r x I V(\boldsymbol{A a})$ and robo $(\boldsymbol{A} \boldsymbol{b})$ mutants, respectively, slit mutants show presence of low level of Slit $(\boldsymbol{A c})$. $\boldsymbol{B}-\boldsymbol{D}$, Wild-type and $n r x I V(\boldsymbol{B}), \operatorname{robo}(\boldsymbol{C})$, and slit (D) mutant embryonic lysates were immunoprecipitated with anti-Nrx IV (B), anti-Robo $(\boldsymbol{C})$, and anti-Slit (D), respectively. Wildtype IPs show the presence of Nrx IV $(\boldsymbol{B} \boldsymbol{a}, \boldsymbol{C} \boldsymbol{b}, \boldsymbol{D b})$, Robo $(\boldsymbol{B} \boldsymbol{b}, \boldsymbol{C} \boldsymbol{a}, \boldsymbol{D C})$, and Slit (BC, $\boldsymbol{C}$, and $\mathbf{D a})$. nrx IV mutant IPs with anti-Nrx IV antibodies do not show the presence of Nrx IV $(\boldsymbol{B a})$, Robo $(\boldsymbol{B} \boldsymbol{b})$, and Slit $(\boldsymbol{B C})$. robo mutant IPs with anti-Robo antibodies do not show the presence of Robo (Ca), Nrx IV (Cb), and Slit (Cc). slit mutant IPs with Slit antibodies show traces of Slit protein (Da) but do not show detectable levels of Nrx IV (Db) or Robo (DC). $\boldsymbol{E}-\mathbf{G}$, IPs from the wild-type and $n r x I V$, robo, and slit mutant embryos show

tibodies do not show presence of Robo and Slit (Fig. $6 B b, B c$ ) thus confirming the specificity of IPs. Similarly, Nrx IV and Slit are present in IP complexes with antiRobo antibodies from the wild-type embryos and not from the robo mutants (Fig. $6 \mathrm{Ca}-\mathrm{Cc}$ ). IPs using anti-Slit antibodies show the presence of Nrx IV and Robo from the wild-type lysates and not from the slit mutants (Fig. $6 D b, D c$ ). Note that some Slit protein is present in IPs from the slit mutants but this does not seem to be sufficient to IP Nrx IV and Robo from slit mutants. The molecular interactions between Nrx IV, Robo, and Slit were also observed in IP complexes with S2 cell extracts (see below). Together, these data strongly indicate that Nrx IV, Robo, and Slit form an in vivo biochemical complex.

Having established that Nrx IV, Robo, and Slit exist in a molecular complex, we next wanted to determine whether loss of any one of these proteins results in the breakdown of this molecular complex in vivo. We performed IPs using embryonic lysates from COPAS-sorted wild-type and $n r x I V$, robo, and slit mutants. As shown in Figure 6, $E a$ and $E b$, loss of Nrx IV does not abolish the binding of Robo and Slit, as Slit is present in the Robo IP complex, although Slit levels in the IP complex are reduced compared with wild-type levels. Similarly, loss of Robo does not abolish the complex formation between Slit and Nrx IV (Fig. 6Fa, Fb), although the levels of Nrx IV are reduced in robo mutants (Fig. $6 \mathrm{Fa}$ ). In slit mutants, loss of Slit does not disrupt interactions between Nrx IV and Robo (Fig. $6 G a, G b$ ). This was further confirmed by using slit RNAi in S2 cells. RNAi against slit showed essentially no detectable Slit protein in S2 cells (Fig. $6 \mathrm{Ha}$, compare lane sli RNAi Lys with lane Con Lys). IPs using anti-Nrx IV antibod-

$\leftarrow$

that loss of any one of these proteins does not abolish the complex formation between the remaining two proteins. (Note the breaks in panels are due to removal of irrelevant lanes). $\boldsymbol{H}$, Equal amounts of control $\mathbf{S 2}$ cells and cells treated with slit dsRNA show presence of Slit in control, while complete lack of Slit in the slit RNAi knock down cells $(\mathrm{Ha})$. The same blot was probed with anti- $\beta$-tubulin as a loading control (Hb). II IPs from control S2 and slit RNAi cells show that in the absence of Slit, Nrx IV, and Robo still associate in a complex, although less efficiently compared with controls. J, Immunoprecipitation from wild-type embryo reveals the presence of Nrx IV and Sdc in the same complex. $K$, Equal amounts of wildtype and sdc ${ }^{10608}$ and Df48 $8^{\text {ubi-Sara }}$ homozygous embryos show the presence of $\mathrm{Sdc}$ protein $(\mathbf{K a})$, unchanged levels of Nrx IV $(\boldsymbol{K} \boldsymbol{b})$, and $\beta$-tubulin as control $(\boldsymbol{K} \boldsymbol{c}) . \boldsymbol{L}, \boldsymbol{M}$, The specificity of $S d \boldsymbol{c}$ antibody is established by IPs using anti-Nrx IV antibodies in the wild-type and $n r x / V$ mutant embryos $(\boldsymbol{L})$ and Robo antibodies in the wild-type and robo mutant embryos $(\boldsymbol{M})$. 

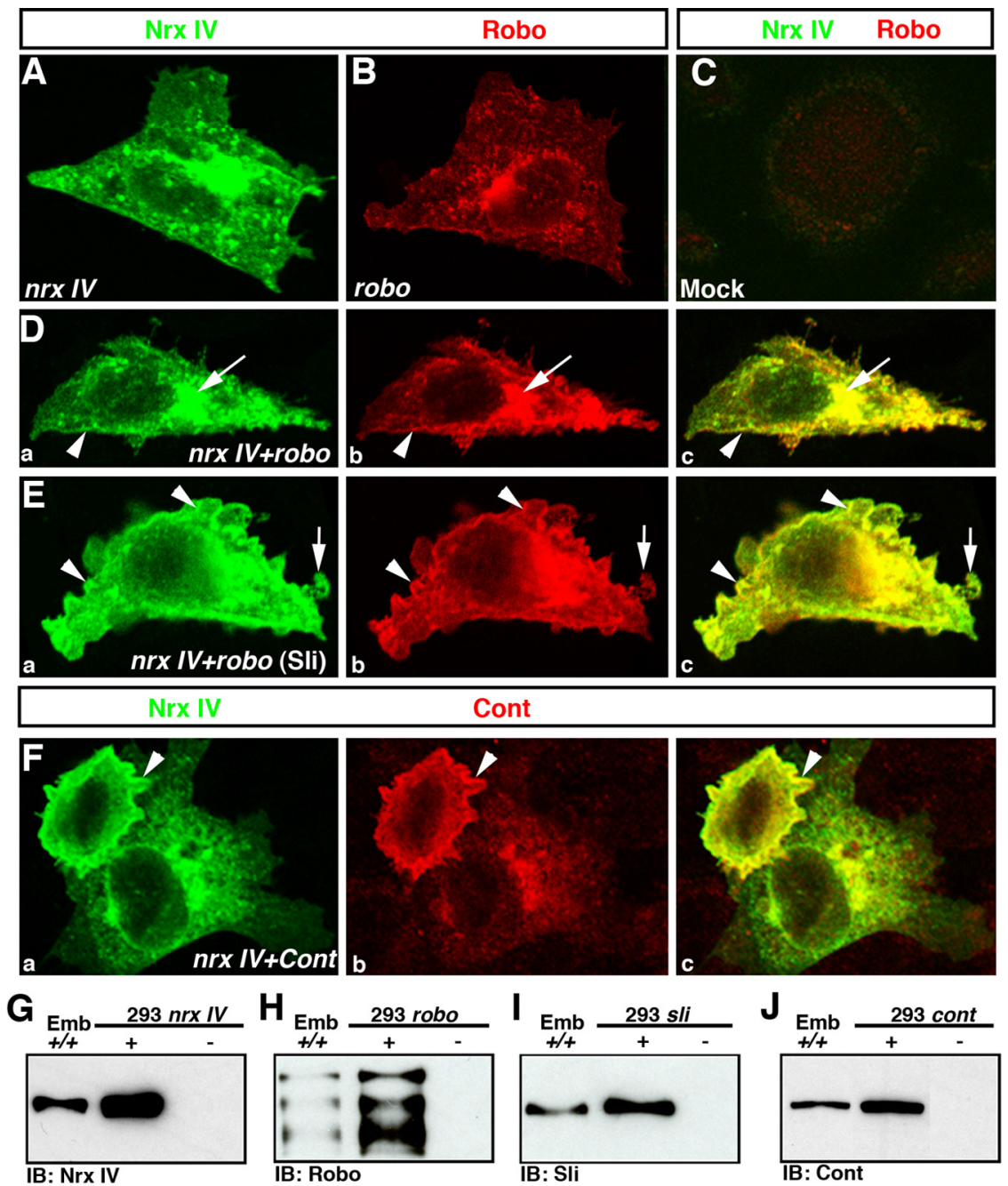

K Sli
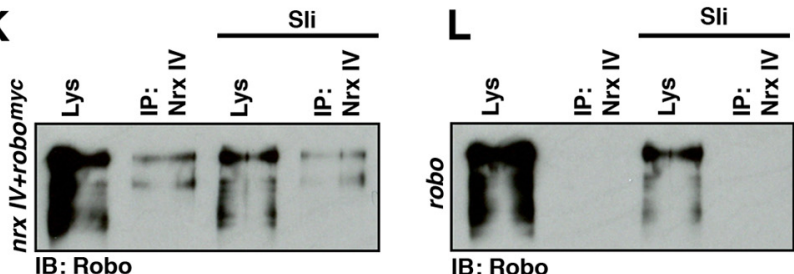

IB: Robo

O

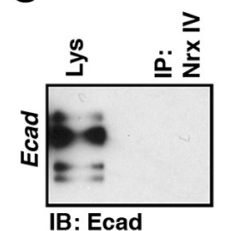

M

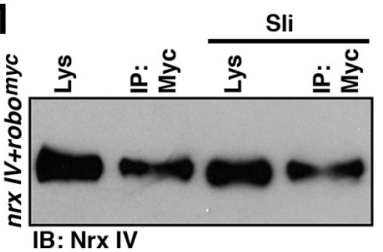

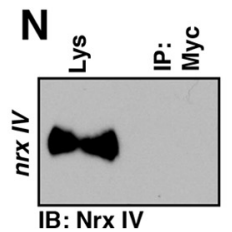

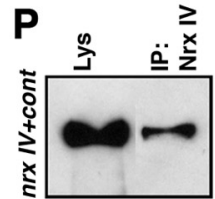

IB: Cont

Figure 7. Nrx IV and Robo interact in a heterologous system. $\boldsymbol{A}, \boldsymbol{B}, \operatorname{nrx} I V(\boldsymbol{A})$ and robo $(\boldsymbol{B})$ localize to both plasma membrane and intracellular compartments in transfected $\mathrm{CHO}_{\mathrm{O}}$ cells. C, Mock-transfected cells stained with Nrx IV and Robo antibodies show undetectable levels of either protein. $\boldsymbol{D}, \boldsymbol{E}$, The distribution of $\operatorname{nrx} I V(\boldsymbol{D a}, \boldsymbol{E a})$ and robo $(\boldsymbol{D b}, \boldsymbol{E b})$ overlap in cotransfected $\mathrm{CHO}$ cells both in the absence $(\boldsymbol{D})$ and presence $(\boldsymbol{E})$ of Slit-conditioned medium. $\boldsymbol{F}$, The distributions of $n r x / V(F \boldsymbol{a})$ and cont $(\boldsymbol{F} \boldsymbol{b})$ also overlap in cotransfected $\mathrm{CHO}$ cells. $\mathbf{G}-\boldsymbol{J}$, Western blot analyses of wild-type embryonic $\left(\mathrm{Emb}^{+/+}\right)$and transfected HEK 293 cells. Cells transfected with $\operatorname{Nrx} I V(\boldsymbol{G})$, Robo $(\boldsymbol{H})$, Slit $(\boldsymbol{I})$, and Cont $(\boldsymbol{J})$ express polypeptides with the same apparent molecular weight seen in embryonic lysates. These bands are not detected in mock-transfected cells $(-), \boldsymbol{K}, \boldsymbol{M}, \mathrm{Nrx}$ IV and Robo ${ }^{\text {myc }}{ }^{\mathrm{c} 0-I \mathrm{IP}}$ from cotransfected HEK 293 cells immunoprecipitated with either anti-Nrx IV $(\boldsymbol{K})$ or anti-Myc $(\boldsymbol{M})$. L, $\boldsymbol{N}, \mathbf{0}$, Control experiments: HEK 293 cells transfected with robo $(\boldsymbol{L}), n r x I V(\boldsymbol{N})$, or Ecad $(\boldsymbol{O})$ and immunoprecipitated with anti-Nrx IV $(\boldsymbol{L}, \mathbf{0})$ or anti-Myc $(\boldsymbol{N})$ antibodies showed no detectable levels of Robo ( $\boldsymbol{L})$, Nrx IV ( $\boldsymbol{N})$, or Ecad ( $\boldsymbol{O}$ ) in IPs. P, Positive control: Cont coprecipitates with Nrx IV in cotransfected HEK 293 cells.

ies with slit RNAi S2 cell extracts showed the presence of reduced levels of Robo (Fig. 6I) compared with the control lysates, indicating that Robo and Nrx IV interact in the absence of Slit. These data suggest that loss of any one of these proteins does not abolish the complex formation between the two remaining proteins and support the idea that the Nrx IV/ Robo/Slit signaling complex may exist as a macromolecular complex with other molecular components.

Since HSPG Sdc is one such protein that exists in a molecular complex with Robo and Slit (Johnson et al., 2004), we wanted to test whether Sdc and Nrx IV are also in the same complex. IP with anti-Sdc from wild-type embryonic lysates showed the presence of Nrx IV (Fig. $6 J$ ). The specificity of Sdc antibody was determined by carrying out IPs with anti-Nrx IV antibodies from wild-type and $n r x I V$ mutants. Sdc was detected only in the wild-type and not in $n r x I V$ mutant lysates (Fig. $6 L$ ). Similarly Sdc was detected in IPs with anti-Robo antibodies from the wildtype and not the robo mutant lysates (Fig. $6 M)$. We also analyzed $s d c$ mutants by IB analysis against Sdc and Nrx IV. As shown in Figure $6 K$, in two independent $s d c$ mutants, Sdc levels were slightly reduced but not absent compared with the wild type because of significant amounts of the maternal Sdc protein (Johnson et al., 2004) (Fig. $6 \mathrm{Ka}$ ). The Nrx IV protein levels were not affected in either $s d c$ mutant (Fig. $6 K b)$. Furthermore, no significant alteration in the subcellular localization of $\mathrm{Nrx}$ IV was observed in $s d c$ mutants (supplemental Fig. S4, available at www. jneurosci.org as supplemental material). Together, the biochemical analyses indicate that Nrx IV is an essential component of the Robo/Slit protein complex and that loss of any of the components does not abolish the formation of the complex but reduces the efficacy of the complex formation between the remaining components.

\section{Nrx IV and Robo colocalize and} interact in a heterologous system The axonal localization (Fig. 3) and in vivo biochemical association (Fig. 6) of Nrx IV and Robo suggest that these proteins interact in cis within the plasma membrane. To further confirm the cis interaction, we examined the subcellular localization and biochemical association of Nrx IV and Robo in a heterologous system. We transfected robo and nrx IV, either separately or together, into mammalian $\mathrm{CHO}$ and HEK 293 cells. Both Nrx IV (Fig. 7A) and Robo (Fig. $7 B$ ) localize to intracellular vesicles 
and the cell membrane in $\mathrm{CHO}$ cells. Little if any staining was detected in mocktransfected cells stained with antibodies against these two proteins (Fig. 7C). In $\mathrm{CHO}$ cells cotransfected with $n r x I V$ (Fig. $7 D a$ ) and robo (Fig. $7 D b$ ), the proteins colocalize within intracellular vesicular compartments (Fig. $7 D a-D c$, arrows) and the cell plasma membrane (Fig. $7 D a-D c$, arrowheads). Since earlier studies have shown that Slit binding to the Robo receptor can recruit cytosolic effectors like PAK and Dock (Wong et al., 2001; Fan et al., 2003), we also examined whether Slit stimulation had any effect on the overlapping distribution of Nrx IV and Robo in cotransfected $\mathrm{CHO}$ cells. As demonstrated in Figure $7 E$, the staining of both Nrx IV (Fig. $7 E a$ ) and Robo (Fig. $7 E b$ ), processed and imaged under identical conditions, shows that the proteins still colocalize within the cell membranes. Interestingly, both proteins also showed increased colocalization in plasma membrane ruffles (Fig. $7 E c$, arrowheads) and membrane extensions such as filopodia (Fig. $7 E a-E c$, arrows). This colocalization is qualitatively similar to that previously observed for Nrx IV and Contactin (Fig. $7 F$ ), two well established binding partners (Faivre-Sarrailh et al., 2004). These data indicate that Nrx IV and Robo retain their ability to colocalize in a nonDrosophila cell system in the absence of other Drosophila proteins.

We next addressed whether Nrx IV and Robo could form a complex in mammalian cells, and whether addition of Slit would enhance the complex formation. Initially, HEK 293 cells were separately transfected with $p c D N A 3.1$ containing $n r x$ $I V$, robo, and slit cDNAs and processed for IB analysis to confirm that the expressed polypeptides were comparable in size to the endogenous proteins from Drosophila embryos. We found that Nrx IV (Fig. 7G), Robo (Fig. 7H), and Slit (Fig. 7I) expressed in 293 cells had the same apparent molecular weight as the correlating embryonic proteins (Fig. 7G-I, Emb), suggesting that the expression of these Drosophila proteins in mammalian cells did not interfere with their processing or post-translational modifications. The same was true for cont transfected 293 cells (a positive control) (Fig. 7J) (Faivre-Sarrailh et al., 2004). Mock-transfected 293 cells did not show detectable levels of any of these Drosophila proteins (Fig. $7 G-J$, lanes marked as -$)$. We next analyzed the interaction between Nrx IV and Robo ${ }^{\text {myc }}$ (Bashaw et al., 2000) in absence and presence of Slit treatment by immunoprecipitating either Nrx IV (Fig. $7 \mathrm{~K}$ ) or Myc (Fig. 7M) from 293 cells cotransfected with both $n r x I V$ and $r o b o^{m y c}$. We found that both Robo and Nrx IV were detected in IPs with anti-Nrx IV (Fig. $7 K$ ) and anti-Myc (Fig. $7 M)$, respectively, confirming that Nrx IV and Robo interact in a heterologous system. Slit treatment of $n r x I V$ - and robo ${ }^{m y c_{-}}$ cotransfected 293 cells did not seem to enhance the coprecipitation of Nrx IV and Robo. We were unable to co-IP Robo (Fig. 7L),

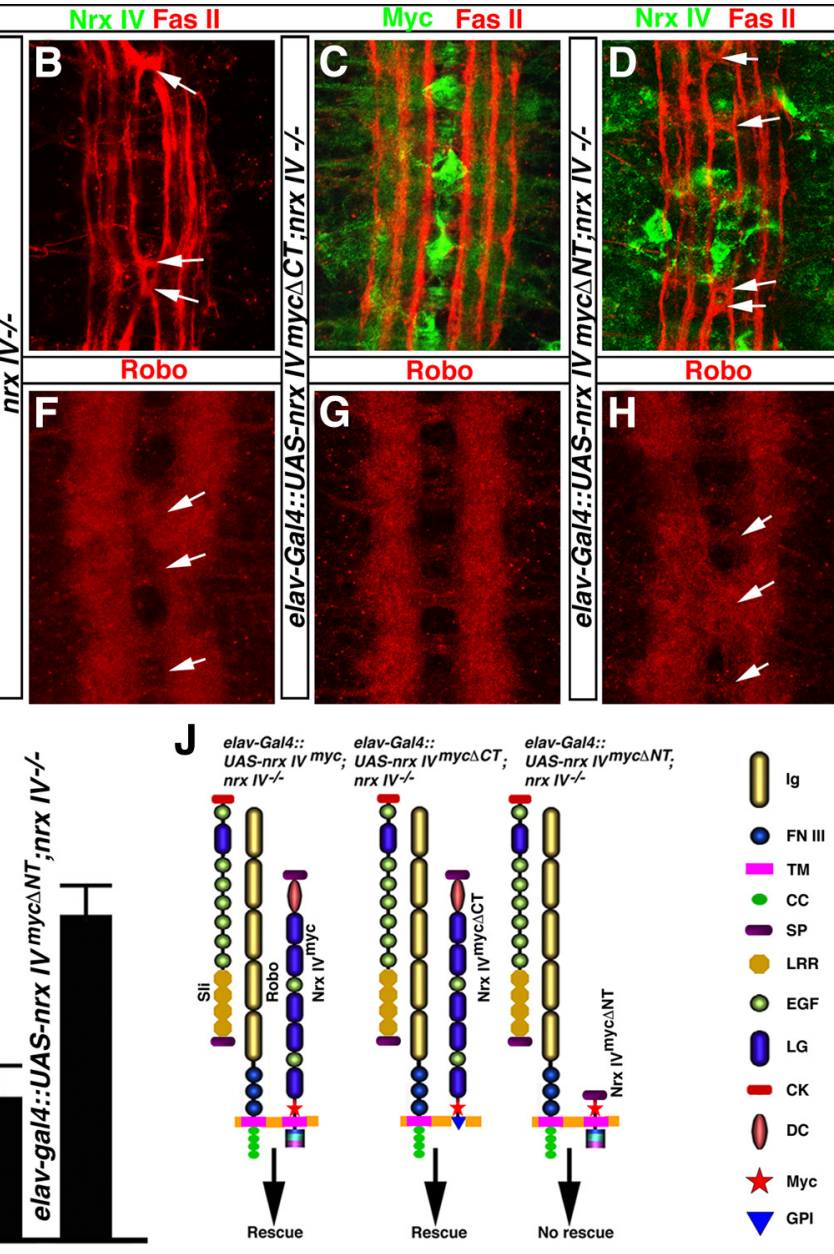

Figure 8. The extracellular domain of $\mathrm{Nrx} I V$ is sufficient to rescue the midline axon guidance phenotype and Robo localization.

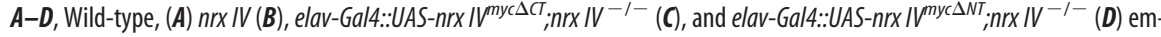
bryos expressing Nrx IV $(\boldsymbol{A}, \boldsymbol{B}, \boldsymbol{D}$, green), Fas II $(\boldsymbol{A}, \boldsymbol{B}, \boldsymbol{D}$, red), and Myc ( $\boldsymbol{C}$, green) and Fas $\|(\boldsymbol{C}$, red). $\boldsymbol{E}-\boldsymbol{H}$, Robo localization in

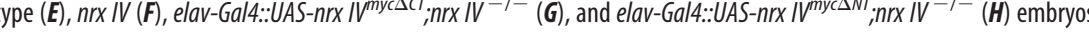

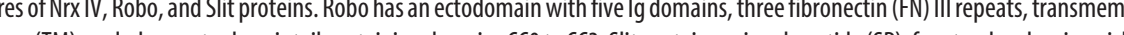
repeats (LRRs), epidermal growth factor (EGF) repeats, a conserved laminin G spacer also found in Nrx IV, laminin, agrin, and perlecan, and a C-terminal cysteine knot (CK). Nrx IV has an SP, discoidin (DC), laminin G (LG), EGF repeats, a TM and a short C-terminal tail. GPI, Glycosylphosphatidylinositol.

Nrx IV (Fig. $7 N$ ), or the adhesion molecule E-cadherin (Ecad) (Fig. 7O) from transfected 293 cells with either Nrx IV (Fig. $7 L, O)$ or Myc (Fig. $7 N$ ) antibodies, indicating that the observed IP of Nrx and Robo was highly specific. However, we were able to co-IP the known binding partners Nrx IV and Cont from $n r x I V$ and cont-cotransfected 293 cells with anti-Nrx IV antibodies (Fig. 7P) (Faivre-Sarrailh et al., 2004). These studies support our in vivo findings by demonstrating that Nrx IV and Robo can form a molecular complex in a heterologous system.

\section{Robo localization and midline axon guidance function requires the extracellular domain of Nrx IV}

The cytoplasmic regions of many transmembrane receptor proteins play key roles in signal transduction by interacting with intracellular proteins. Since Robo is a transmembrane receptor for Slit and colocalizes and biochemically interacts with Nrx IV, we wanted to know whether Nrx IV plays a direct role as a signal transducer independent of or alongside Robo. Nrx IV being a transmembrane protein with a 
large extracellular region and a short cytoplasmic domain, we set out to determine whether Nrx IV lacking the cytoplasmic domain would be able to rescue the axon guidance phenotype. To accomplish this, we generated two independent transgenic fly lines with UAS constructs that expressed myc-tagged truncations of Nrx IV protein (Fig. $8 \mathrm{~J}$ ) in which either the transmembrane and the intracellular region was replaced with a glycosylphosphatidylinositol anchor $\left(\mathrm{Nrx} \mathrm{IV}^{\mathrm{myc} \Delta \mathrm{CT}}\right)$ or the extracellular region was deleted (Nrx $\left.\mathrm{IV}^{\mathrm{myc} \Delta \mathrm{NT}}\right)$. In wild-type embryos, Nrx IV localization is in the CNS (Fig. 1) and Fas II expression is seen in ipsilaterally projecting axons (Fig. 8A). Robo localization is at high levels in the longitudinally projecting axons and low in the commissural tracts (Fig. 8E). nrx IV mutants show complete loss of Nrx IV protein as expected and the typical Fas II axonal phenotype in the ML (Fig. $8 B$, arrows). Robo localization in $n r x I V$ mutants is diffused and Robo-positive axons are found to cross and collapse at the ML (Fig. $8 F$, arrows). Expression of UAS-nrx $I V^{m y c \Delta C T}$ with elav-Gal4 in $n r x I V$ mutants significantly rescued the Fas II crossing as well as Robo localization phenotypes (Fig. 8C,G, respectively; Fig. 8I) compared with homozygous nrx IV mutants (Fig. $8 B, F)$. Expression of elav-Gal4::UAS-nrx IV $V^{m y c \Delta N T}$ in $n r x I V$ mutants failed to rescue either the Fas II axon crossing (Fig. $8 D$, arrows) or the Robo localization phenotypes (Fig. $8 H$, arrows; Fig. 8I). These data indicate that the formation of the Nrx IV/ Robo/Slit complex and the axon guidance function of Nrx IV reside in the extracellular region of the protein and that the cytoplasmic region is dispensable for this function. These data also suggest that Nrx IV might not act as a signal transducer but rather functions to allow proper localization and stability of Robo and formation of a macromolecular signaling complex that involves Nrx IV, Robo, Slit, and other associated proteins for efficient repulsive ML axon guidance.

\section{Discussion}

Here, we have provided in vivo genetic, cell biological, and biochemical evidence that Nrx IV functions in ML repulsion of axons and through its interactions with Robo to ensure proper Robo localization in the embryonic CNS. Furthermore, we show that Nrx IV and Robo retain their ability to colocalize and interact in a heterologous cell system, reaffirming their functional interactions. Together, these data provide new insights into the additional roles of Nrx IV outside the realm of SJs and identify Nrx IV as an important interacting partner of the Slit/Robo signaling complex during ML repulsion.

\section{Varied patterns of Nrx IV localization in the CNS underscore different functions}

Recent studies from our laboratory and others have established strong expression of Nrx IV at the interface between neurons and MG that underlies the adhesive interactions between these cells in maintaining ML cytoarchitecture. This function of Nrx IV is Wrapper dependent (Stork et al., 2009; Wheeler et al., 2009). We believe that the axon guidance function of Nrx IV is Wrapper independent based on the following observations. First, the juxtaposition of strong neuronal Nrx IV and glial Wrapper in the ML, together with the absence of axon guidance phenotype in wrapper mutants (Fig. 2) strongly suggests that Nrx IV localization in ML neurons does not contribute to the axon guidance function of Nrx IV. This is further supported by the failure to rescue the axon guidance phenotypes in $n r x I V$ mutant by expressing Nrx IV in all ML neurons and glia with sim-Gal4::UAS-nrx IV (Fig. 1). Therefore, a significant contribution of the axon guidance phenotype seen in nrx IV mutants is unlikely to come from ML neurons or glia. Interestingly, the MG ensheathment defects seen in $n r x I V$ mutants are rescued by expression of Nrx IV in neurons alone and not MG (Wheeler et al., 2009). Furthermore, no changes in Robo localization or crossing of Robo-positive axons were seen in wrapper mutants, thus providing additional evidence that alterations in MG/neuronal architecture in wrapper mutants does not significantly contribute to the axon guidance phenotype (data not shown). Of note, older stage 16 robo and slit mutants show considerable disorganization of MG, as revealed by immunostaining with antiWrapper antibodies (Fig. 5); however, these glial phenotypes are thought to be secondary to their axon guidance phenotypes. In addition, Cont and Nrg, two well established binding partners of Nrx IV at SJs, do not contribute to Nrx IV in its CNS axon guidance function, as revealed by Fas II immunostaining of cont and $n r g$ mutants (supplemental Fig. S2, available at www.jneurosci. org as supplemental material). Based on these observations, we believe that Nrx IV is a multifunctional protein that functions in a cell-type-specific manner. Therefore, the axon guidance function of Nrx IV is to ensure proper localization and stability of Robo in the lateral CNS soma and axons during ML axon repulsion.

Additional support for tissue-specific functions of Nrx IV comes from recent reports that Drosophila cardiac development uses a noncanonical role of Nrx IV to maintain cardiac integrity, by coupling with G-protein signaling (Yi et al., 2008). Both Robo and Slit have previously been shown to control cardiac cell polarity and morphogenesis (Qian et al., 2005; Santiago-Martínez et al., 2008). Since embryonic heart cells lack SJs, these findings further underscore the fact that Nrx IV and Robo/Slit coordinate diverse roles in different tissues involving multiple molecular partners. Thus, Nrx IV functions in Slit/Robo axon guidance pathway independently of its other known partners, such as Cont, Nrg, and Wrapper.

\section{Molecular complex of Nrx IV, Robo, and Slit: direct and indirect interactions}

Our findings strongly support the existence of Nrx IV, Robo, and Slit as a molecular complex. Although Nrx IV function appears interwoven with Robo and Slit, the phenotypes displayed by $n r x$ $I V$ mutants do not completely phenocopy either slit or robo mutants, suggesting that Nrx IV plays a modulatory role in Slit/Robo signaling. The biochemical analyses suggest that Robo is required for Nrx IV stability, as the levels of Nrx IV are significantly reduced in robo mutants (Fig. $6 A, F$ ). Slit, on the other hand, showed a modest stimulatory effect on Robo and Nrx IV association and expression levels, further confirming that these three proteins are functionally interlinked. Furthermore, the Slit/Robo complex is less efficiently immunoprecipitated from $n r x I V$ mutants (Fig. $6 E$ ). Thus, while loss of Nrx IV does not abolish interactions between Robo and Slit, it could potentially affect proper functioning of the Robo/Slit signaling complex. Similarly, reduced association of Slit and Nrx IV in robo mutants suggests that Robo is also important for efficient complex formation between these three proteins.

Both Nrx IV and Robo are transmembrane proteins that colocalize in longitudinal axons. Most of the known Nrx IVinteracting proteins, such as Cont, Nrg, and Wrapper, belong to Ig superfamily of cell adhesion molecules (CAMs) (TessierLavigne and Goodman, 1996). Therefore, it is conceivable that Nrx IV associates with Robo (an Ig CAM) in neurons that do not express detectable levels of Cont or Wrap. Furthermore, $n r x I V$ mutant phenotypes resemble those of robo mutants (Fig. 1 Jb), and Nrx IV interacts with Robo/Slit, suggesting that Nrx IV functions in the Robo/Slit pathway. 
Recent studies support a model in which Slit stimulation recruits cytoplasmic Sos to Robo receptor via Dock to activate Racdependent cytoskeletal changes within the growth cone during repulsion (Yang and Bashaw, 2006). We show that Nrx IV and Robo retain their ability to colocalize and interact when coexpressed in a heterologous system and indicate that Slit is dispensable for their interaction. In addition, slit RNAi experiments in S2 cells reveal that Nrx IV and Robo associate in the absence of Slit. However, Slit stimulation of $n r x$ IV/robo cotransfected CHO cells caused enhanced colocalization of Nrx IV and Robo in intracellular compartments and membrane ruffles, further supporting a functional relationship between Nrx IV and Robo/Slit (Fig. 7). Together, our in vivo and in vitro findings indicate that Nrx IV and Robo interact in the absence of Slit, and in the presence of Slit ligand the molecular interactions between Nrx IV and Robo are strengthened. Formation of this larger molecular complex at the axonal surface thus ensures proper ML axon guidance.

\section{Nrx IV: modulator of Robo/Slit signaling}

The phenotypic similarities, dose-dependent genetic interactions, and in vivo biochemical data suggest that Nrx IV acts as a modulator in Slit/Robo signaling pathway. One of the key reasons for this conclusion is the fact that the axon guidance phenotypes in $n r x I V$ mutants are rescued by the expression of the extracellular region of $\mathrm{Nrx} I V\left(\mathrm{Nrx} \mathrm{IV}^{\mathrm{myc} \Delta \mathrm{CT})}\right.$, and this phenotype is not rescued by the intracellular region of $\mathrm{Nrx} I V\left(\mathrm{Nrx} \mathrm{IV}^{\mathrm{myc} \Delta \mathrm{NT}}\right)$. For Nrx IV to act as an independent signal transducer, it would need an intact cytoplasmic region. Since the axon guidance phenotypes and Robo localization (Fig. 8) are both rescued by Nrx $\mathrm{IV}^{\mathrm{myc} \Delta \mathrm{CT}}$, the downstream signaling controlling axon repulsion is controlled by Robo or an as-yet-unidentified protein. Therefore, our data support a role for Nrx IV in the proper localization and stabilization of Robo at axonal membrane, where it interacts with Slit, to regulate downstream axon guidance signaling. Although the exact domains regulating Nrx IV-Robo interactions are unknown at this point, we predict that they occur via the Ig or FNIII domains, as these domains regulate Nrx IV-Cont interactions. Our data rule out the possibility that Nrx IV interactions with Robo occur via a large cytoskeletal scaffolding complex, as Nrx IV ${ }^{\text {myc } \Delta C T}$ lacks the cytoplasmic region. This allows us to conclude that Nrx IV and Robo interact in cis through their extracellular regions and therefore to eliminate the possibility of a parallel Nrx IV signaling pathway.

One of the interesting in vivo observations is the association between Nrx IV and Slit still occurs in robo-null mutant embryos, indicating that Slit/Nrx IV can interact in the absence of Robo. Although it remains to be seen whether Nrx IV and Slit can associate in the absence of all Drosophila proteins in a heterologous system, based on the existing findings it is tempting to speculate that Nrx IV may function as a coreceptor for Slit, and together with Robo, they stabilize the complex to ensure proper presentation or retention at the axonal surface. A similar role has been assigned to Sdc, which is thought to be critical for the fidelity of Slit repellent signaling, as $s d c$ mutants exhibit consistent defects in ML axon guidance (Johnson et al., 2004; Steigemann et al., 2004; Furrer et al., 2007). Thus, a multitude of mechanisms seem to operate at the axonal surface and growth cones to ensure that axons reach their correct targets (Spitzweck et al., 2010). The Slit-independent interactions between Nrx IV and Robo, but seemingly enhanced colocalization and interactions in the presence of Slit, point to an interesting mechanism whereby signaling molecules use accessory proteins to ensure their proper localization and stability. This mechanism ensures checks and balances at several molecular levels to allow navigating axons to reach their final destinations. To our knowledge, very few proteins have been implicated in Slit/Robo signaling at the axonal surface, and additional yet-unidentified proteins may be involved. With the identification of Nrx IV as an essential component of the Slit/Robo complex, new insights into this highly sophisticated molecular pathway are opened, and this may allow future studies aimed at identifying the modulatory proteins that coordinate and/or control axon guidance.

\section{References}

Andrews GL, Tanglao S, Farmer WT, Morin S, Brotman S, Berberoglu MA, Price H, Fernandez GC, Mastick GS, Charron F, Kidd T (2008) Dscam guides embryonic axons by Netrin-dependent and -independent functions. Development 135:3839-3848.

Banerjee S, Pillai AM, Paik R, Li J, Bhat MA (2006) Axonal ensheathment and septate junction formation in the peripheral nervous system of Drosophila. J Neurosci 26:3319-3329.

Banerjee S, Bainton RJ, Mayer N, Beckstead R, Bhat MA (2008) Septate junctions are required for ommatidial integrity and blood-eye barrier function in Drosophila. Dev Biol 317:585-599.

Bashaw GJ, Kidd T, Murray D, Pawson T, Goodman CS (2000) Repulsive axon guidance: Abelson and Enabled play opposing roles downstream of the roundabout receptor. Cell 101:703-715.

Battye R, Stevens A, Jacobs JR (1999) Axon repulsion from the midline of the Drosophila CNS requires slit function. Development 126:2475-2481.

Baumgartner S, Littleton JT, Broadie K, Bhat MA, Harbecke R, Lengyel JA, Chiquet-Ehrismann R, Prokop A, Bellen HJ (1996) A Drosophila neurexin is required for septate junction and blood-nerve barrier formation and function. Cell 87:1059-1068.

Brand A (1995) GFP in Drosophila. Trends Genet 11:324-325.

Brankatschk M, Dickson BJ (2006) Netrins guide Drosophila commissural axons at short range. Nat Neurosci 9:188-194.

Brose K, Bland KS, Wang KH, Arnott D, Henzel W, Goodman CS, TessierLavigne M, Kidd T (1999) Slit proteins bind Robo receptors and have an evolutionarily conserved role in repulsive axon guidance. Cell 96:795-806.

Buszczak M, Paterno S, Lighthouse D, Bachman J, Planck J, Owen S, Skora AD, Nystul TG, Ohlstein B, Allen A, Wilhelm JE, Murphy TD, Levis RW, Matunis E, Srivali N, Hoskins RA, Spradling AC (2007) The Carnegie protein trap library: a versatile tool for Drosophila developmental studies. Genetics 175:1505-1531.

Chanana B, Steigemann P, Jäckle H, Vorbrüggen G (2009) Reception of Slit requires only the chondroitin-sulphate-modified extracellular domain of Syndecan at the target cell surface. Proc Natl Acad Sci USA 106:11984-11988.

Faivre-Sarrailh C, Banerjee S, Li J, Hortsch M, Laval M, Bhat MA (2004) Drosophila contactin, a homolog of vertebrate contactin, is required for septate junction organization and paracellular barrier function. Development 131:4931-4942.

Fan X, Labrador JP, Hing H, Bashaw GJ (2003) Slit stimulation recruits Dock and Pak to the roundabout receptor and increases Rac activity to regulate axon repulsion at the CNS midline. Neuron 40:113-127.

Fritz JL, VanBerkum MF (2000) Calmodulin and son of sevenless dependent signaling pathways regulate midline crossing of axons in the Drosophila CNS. Development 127:1991-2000.

Fritz JL, VanBerkum MF (2002) Regulation of rho family GTPases is required to prevent axons from crossing the midline. Dev Biol 252:46-58.

Furlong EE, Profitt D, Scott MP (2001) Automated sorting of live transgenic embryos. Nat Biotechnol 19:153-156.

Furrer MP, Vasenkova I, Kamiyama D, Rosado Y, Chiba A (2007) Slit and Robo control the development of dendrites in Drosophila CNS. Development 134:3795-3804.

Garbe DS, Bashaw GJ (2004) Axon guidance at the midline: from mutants to mechanisms. Crit Rev Biochem Mol Biol 39:319-341.

Garbe DS, Bashaw GJ (2007) Independent functions of Slit-Robo repulsion and Netrin-Frazzled attraction regulate axon crossing at the midline in Drosophila. J Neurosci 27:3584-3592.

Goodman CS, Doe CQ (1993) Embryonic development of the Drosophila central nervous system. In: The development of Drosophila melanogaster (Bate M, Martinez Arias A, eds), pp 1131-1206. New York: CSHL. 
Harris R, Sabatelli LM, Seeger MA (1996) Guidance cues at the Drosophila CNS midline: identification and characterization of two Drosophila Netrin/UNC-6 homologs. Neuron 17:217-228.

Jacobs JR (2000) The midline glia of Drosophila: a molecular genetic model for the developmental functions of glia. Prog Neurobiol 62:475-508.

Johnson KG, Ghose A, Epstein E, Lincecum J, O'Connor MB, Van Vactor D (2004) Axonal heparan sulfate proteoglycans regulate the distribution and efficiency of the repellent slit during midline axon guidance. Curr Biol 14:499-504.

Keleman K, Dickson BJ (2001) Short- and long-range repulsion by the Drosophila Unc5 netrin receptor. Neuron 32:605-617.

Keleman K, Rajagopalan S, Cleppien D, Teis D, Paiha K, Huber LA, Technau GM, Dickson BJ (2002) Comm sorts robo to control axon guidance at the Drosophila midline. Cell 110:415-427.

Keleman K, Ribeiro C, Dickson BJ (2005) Comm function in commissural axon guidance: cell-autonomous sorting of Robo in vivo. Nat Neurosci $8: 156-163$.

Kidd T, Brose K, Mitchell KJ, Fetter RD, Tessier-Lavigne M, Goodman CS, Tear G (1998) Roundabout controls axon crossing of the CNS midline and defines a novel subfamily of evolutionarily conserved guidance receptors. Cell 92:205-215.

Kidd T, Bland KS, Goodman CS (1999) Slit is the midline repellent for the robo receptor in Drosophila. Cell 96:785-794.

Klämbt C, Jacobs JR, Goodman CS (1991) The midline of the Drosophila central nervous system: a model for the genetic analysis of cell fate, cell migration, and growth cone guidance. Cell 64:801-815.

Kolodziej PA, Timpe LC, Mitchell KJ, Fried SR, Goodman CS, Jan LY, Jan YN (1996) frazzled encodes a Drosophila member of the DCC immunoglobulin subfamily and is required for CNS and motor axon guidance. Cell 87:197-204

Lundgren SE, Callahan CA, Thor S, Thomas JB (1995) Control of neuronal pathway selection by the Drosophila LIM homeodomain gene apterous. Development 121:1769-1773.

Mitchell KJ, Doyle JL, Serafini T, Kennedy TE, Tessier-Lavigne M, Goodman CS, Dickson BJ (1996) Genetic analysis of Netrin genes in Drosophila: Netrins guide CNS commissural axons and peripheral motor axons. Neuron 17:203-215.

Noordermeer JN, Kopczynski CC, Fetter RD, Bland KS, Chen WY, Goodman CS (1998) Wrapper, a novel member of the Ig superfamily, is expressed by midline glia and is required for them to ensheath commissural axons in Drosophila. Neuron 21:991-1001.

O’Donnell M, Chance RK, Bashaw GJ (2009) Axon growth and guidance: receptor regulation and signal transduction. Annu Rev Neurosci 32: 383-412.

Qian L, Liu J, Bodmer R (2005) Slit and Robo control cardiac cell polarity and morphogenesis. Curr Biol 15:2271-2278.
Rajagopalan S, Vivancos V, Nicolas E, Dickson BJ (2000) Selecting a longitudinal pathway: Robo receptors specify the lateral position of axons in the Drosophila CNS. Cell 103:1033-1045.

Rogers SL, Rogers GC (2008) Culture of Drosophila S2 cells and their use for RNAi-mediated loss-of-function studies and immunofluorescence microscopy. Nat Protoc 3:606-611.

Rothberg JM, Jacobs JR, Goodman CS, Artavanis-Tsakonas S (1990) slit: an extracellular protein necessary for development of midline glia and commissural axon pathways contains both EGF and LRR domains. Genes Dev 4:2169-2187.

Santiago-Martínez E, Soplop NH, Patel R, Kramer SG (2008) Repulsion by Slit and Roundabout prevents Shotgun/E-cadherin-mediated cell adhesion during Drosophila heart tube lumen formation. J Cell Biol 182: 241-248.

Seeger M, Tear G, Ferres-Marco D, Goodman CS (1993) Mutations affecting growth cone guidance in Drosophila: genes necessary for guidance toward or away from the midline. Neuron 10:409-426.

Simpson JH, Bland KS, Fetter RD, Goodman CS (2000) Short-range and long-range guidance by Slit and its Robo receptors: a combinatorial code of Robo receptors controls lateral position. Cell 103:1019-1032.

Spitzweck B, Brankatschk M, Dickson BJ (2010) Distinct protein domains and expression patterns confer divergent axon guidance functions for Drosophila Robo receptors. Cell 140:409-420.

Steigemann P, Molitor A, Fellert S, Jäckle H, Vorbrüggen G (2004) Heparan sulfate proteoglycan syndecan promotes axonal and myotube guidance by slit/robo signaling. Curr Biol 14:225-230.

Stork T, Thomas S, Rodrigues F, Silies M, Naffin E, Wenderdel S, Klämbt C (2009) Drosophila Neurexin IV stabilizes neuron-glia interactions at the CNS midline by binding to Wrapper. Development 136:1251-1261.

Tessier-Lavigne M, Goodman CS (1996) The molecular biology of axon guidance. Science 274:1123-1133.

Wheeler SR, Banerjee S, Blauth K, Rogers SL, Bhat MA, Crews ST (2009) Neurexin IV and wrapper interactions mediate Drosophila midline glial migration and axonal ensheathment. Development 136:1147-1157.

Wong K, Ren XR, Huang YZ, Xie Y, Liu G, Saito H, Tang H, Wen L, BradyKalnay SM, Mei L, Wu JY, Xiong WC, Rao Y (2001) Signal transduction in neuronal migration: roles of GTPase activating proteins and the small GTPase Cdc42 in the Slit-Robo pathway. Cell 107:209-221.

Yang L, Bashaw GJ (2006) Son of sevenless directly links the Robo receptor to rac activation to control axon repulsion at the midline. Neuron 52:595-607.

Yang L, Garbe DS, Bashaw GJ (2009) A frazzled/DCC-dependent transcriptional switch regulates midline axon guidance. Science 324:944-947.

Yi P, Johnson AN, Han Z, Wu J, Olson EN (2008) Heterotrimeric G proteins regulate a noncanonical function of septate junction proteins to maintain cardiac integrity in Drosophila. Dev Cell 15:704-713. 